\title{
Ionothermal Synthesis of Uranyl Vanadate Nanoshell
}

\section{Heteropolyoxometalates}

Tsuyoshi A. Kohlgruber, ${ }^{1}$ Ganna A. Senchyk, ${ }^{1}$ Virginia G. Rodriguez, ${ }^{1}$ Stephanie A. Mackley, ${ }^{1}$ Fabrice Dal Bo, ${ }^{1}$ Sergey M. Aksenov, ${ }^{1}$ Jennifer E. S. Szymanowski, ${ }^{1}$ Ginger E. Sigmon, ${ }^{1}$ Allen G. Oliver, ${ }^{2}$ and Peter C. Burns $^{1,2 *}$

${ }^{1}$ Department of Civil and Environmental Engineering and Earth Sciences, University of Notre Dame, Notre Dame, IN, 46556, USA.

${ }^{2}$ Department of Chemistry and Biochemistry, University of Notre Dame, Notre Dame, IN, 46556, USA. 
Figure S1. Optical images of $\left\{U_{8} V_{6}\right\}$-monomer, $\left\{U_{8} V_{6}\right\}$-dimer, $\left\{U_{8} V_{6}\right\}$-chain, and $\left\{U_{6} V_{3}\right\}$ under transmitted light.

$\left\{U_{8} V_{6}\right\}$-monomer yellow acicular crystals

$\left\{U_{8} V_{6}\right\}$-chain yellow fractured blocky crystals

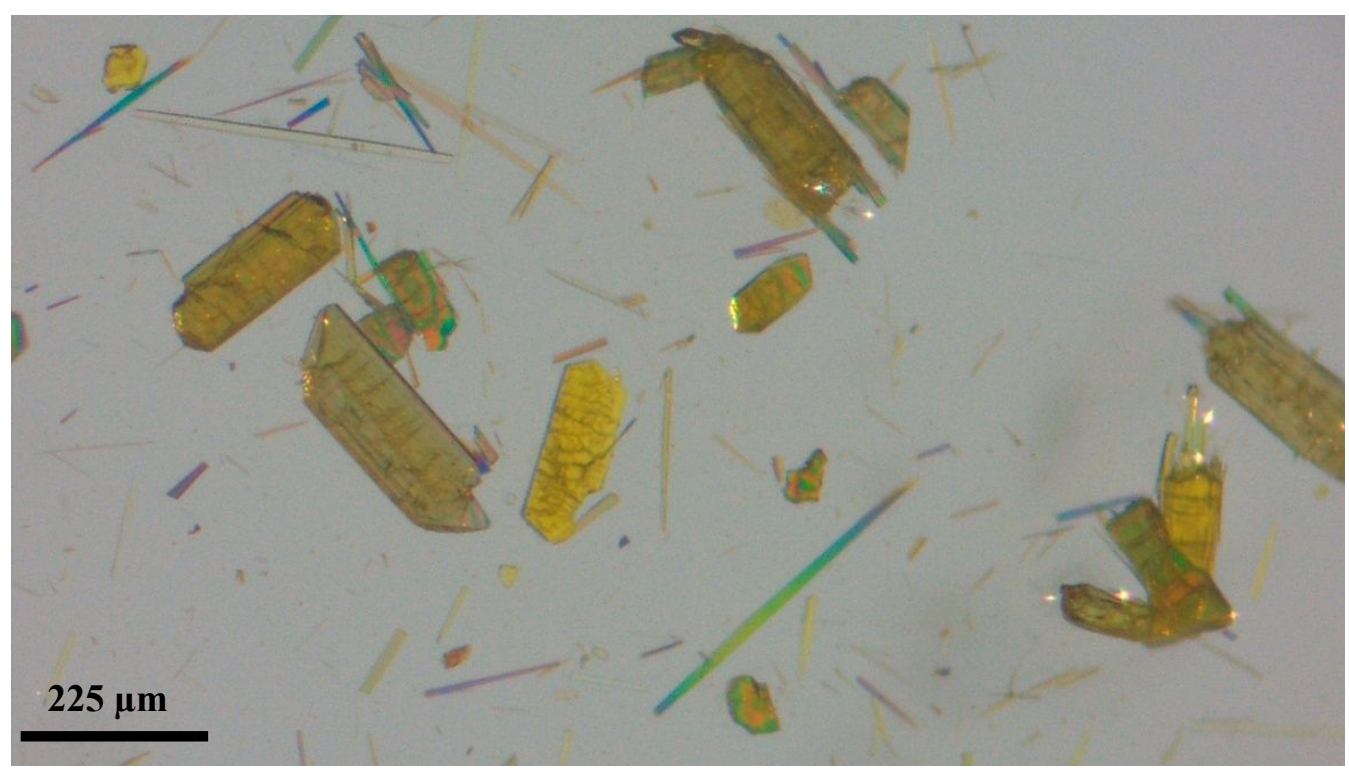

$\left\{U_{\delta} V_{6}\right\}$-dimer yellow crystals among dark-colored byproducts

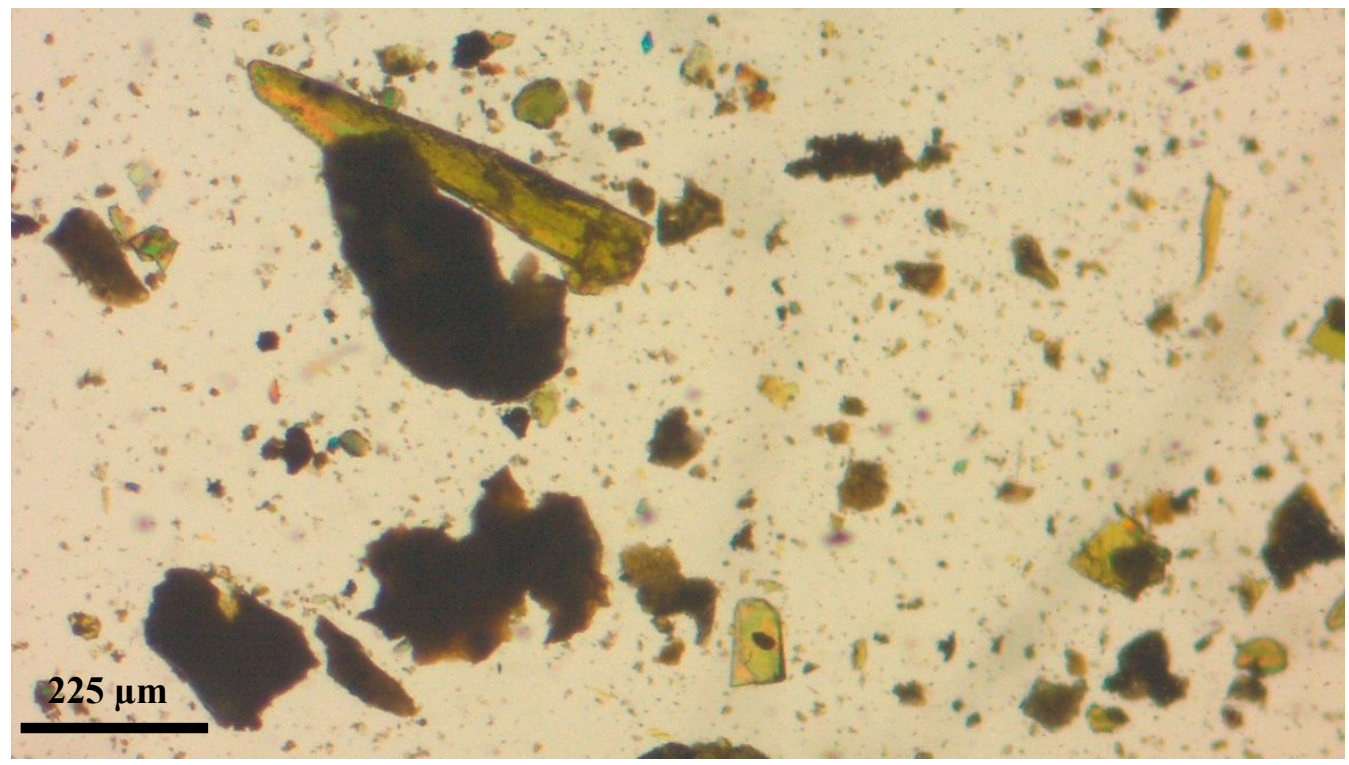


$\left\{U_{6} V_{3}\right\}$ yellow blade-like crystals among dark-colored byproducts

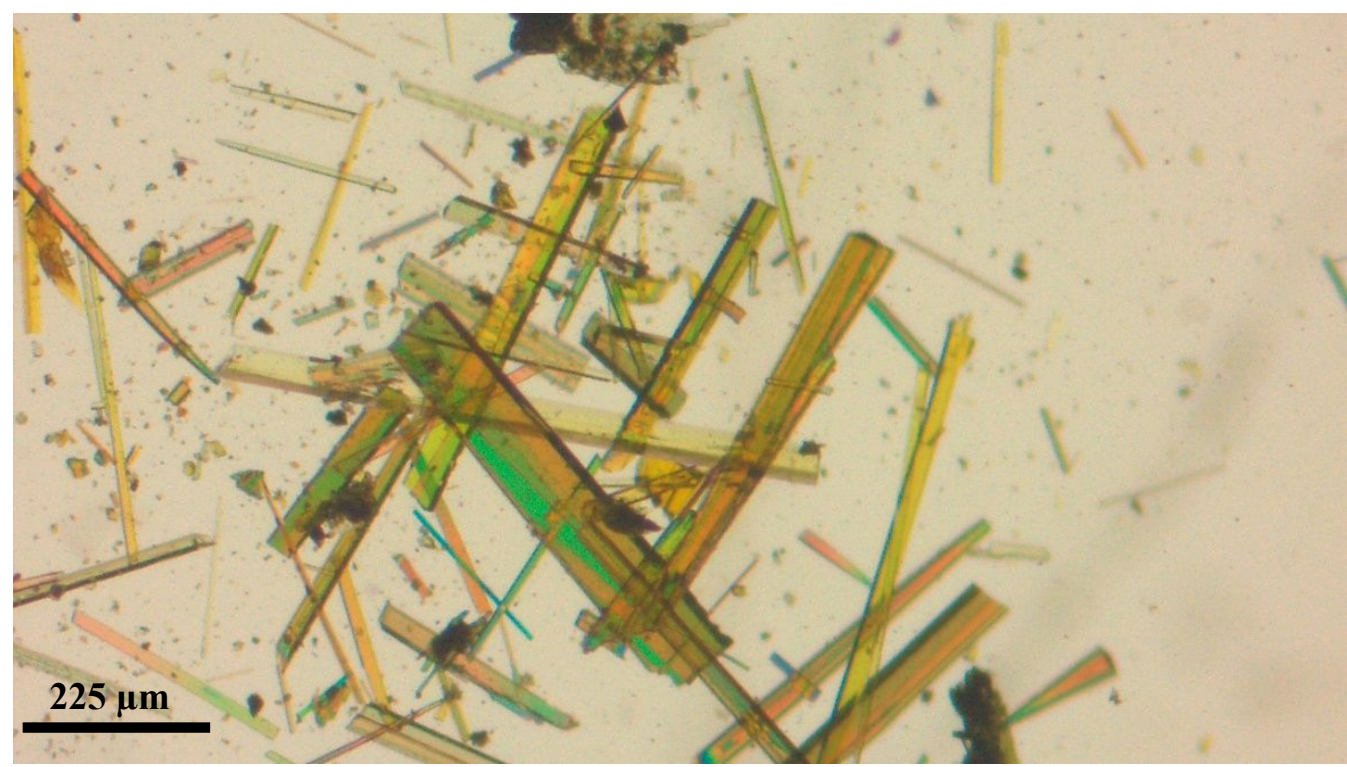


Figure S2. Scanning electron microscopy (SEM) images obtained using a secondary electron detector (SED) for crystals of $\left\{U_{8} V_{6}\right\}$-monomer, $\left\{U_{8} V_{6}\right\}$-dimer, $\left\{U_{8} V_{6}\right\}$-chain, and $\left\{U_{6} V_{3}\right\}$. The corresponding energy dispersive spectra (EDS) of $\left\{U_{8} V_{6}\right\}$-monomer, $\left\{U_{8} V_{6}\right\}$-dimer, $\left\{U_{8} V_{6}\right\}$-chain, and $\left\{\boldsymbol{U}_{6} \boldsymbol{V}_{3}\right\}$ are next to the SEM images.

\section{$\left\{U_{8} V_{6}\right\}$-monomer}
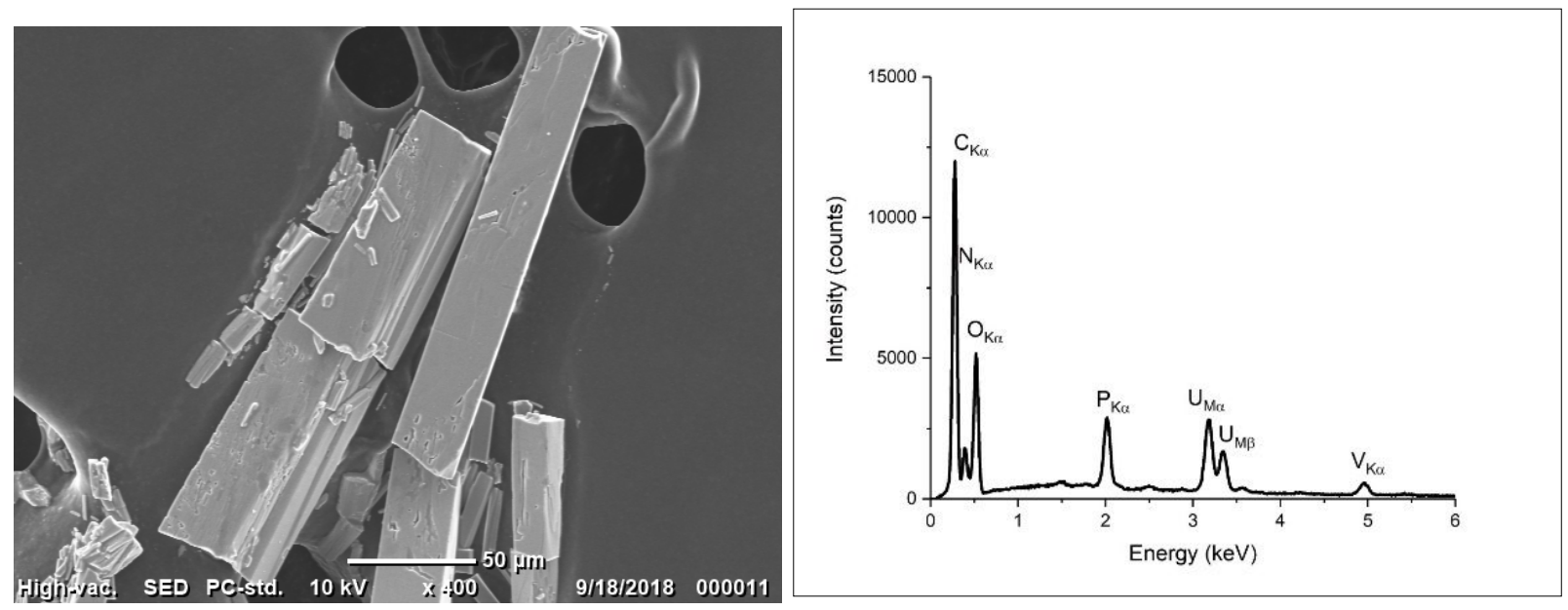

\section{$\left\{U_{8} V_{6}\right\}$-dimer}
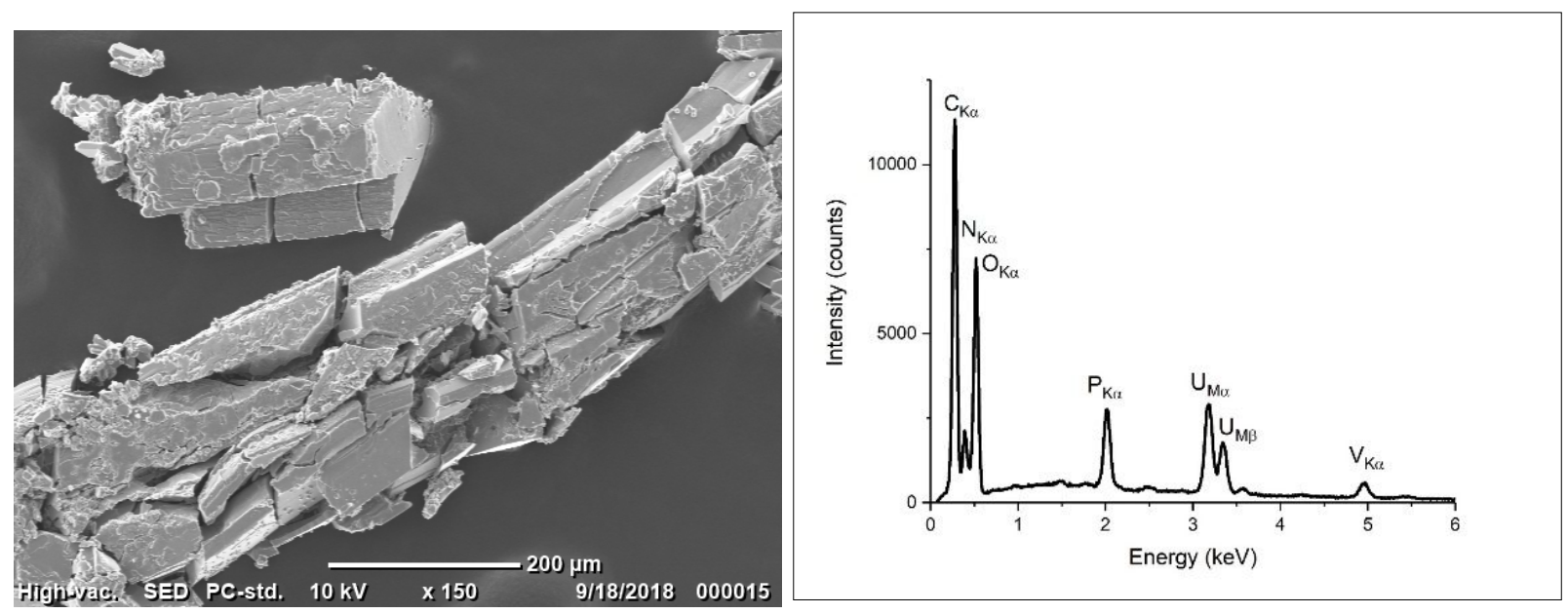


\section{$\left\{U_{8} V_{6}\right\}$-chain}
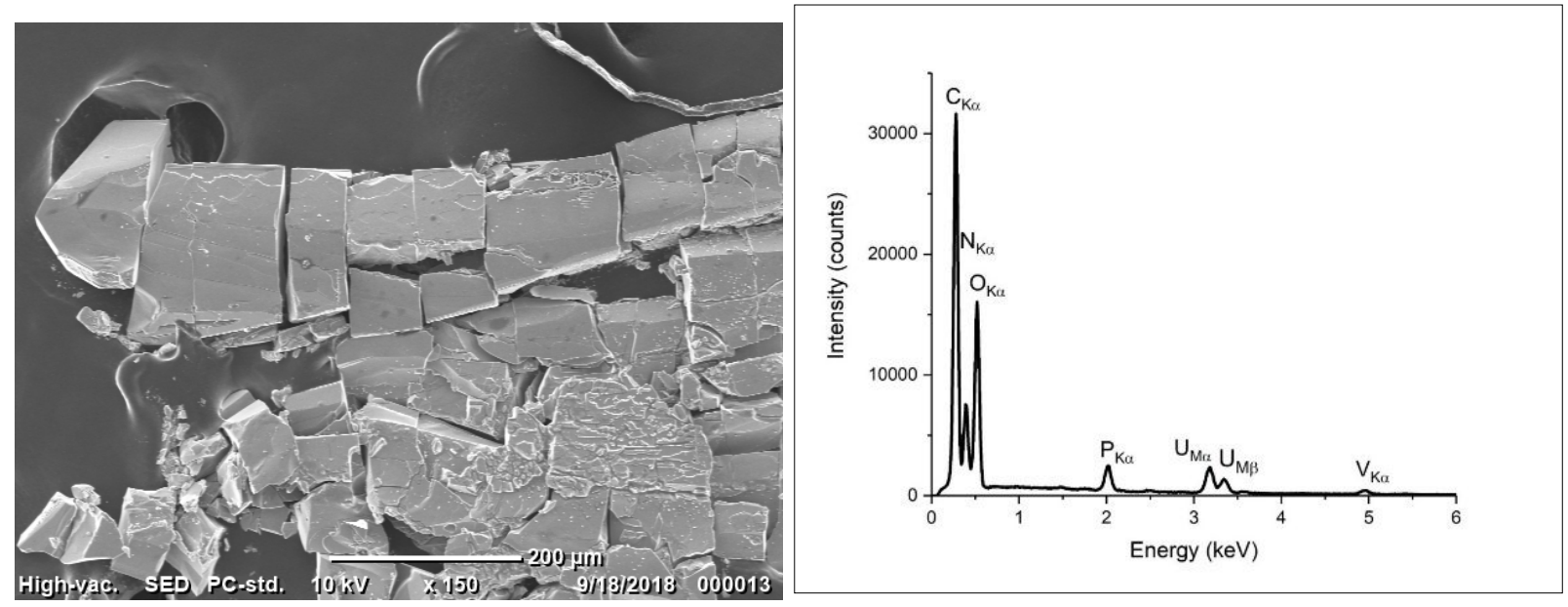

$\left\{\boldsymbol{U}_{6} \boldsymbol{V}_{3}\right\}$
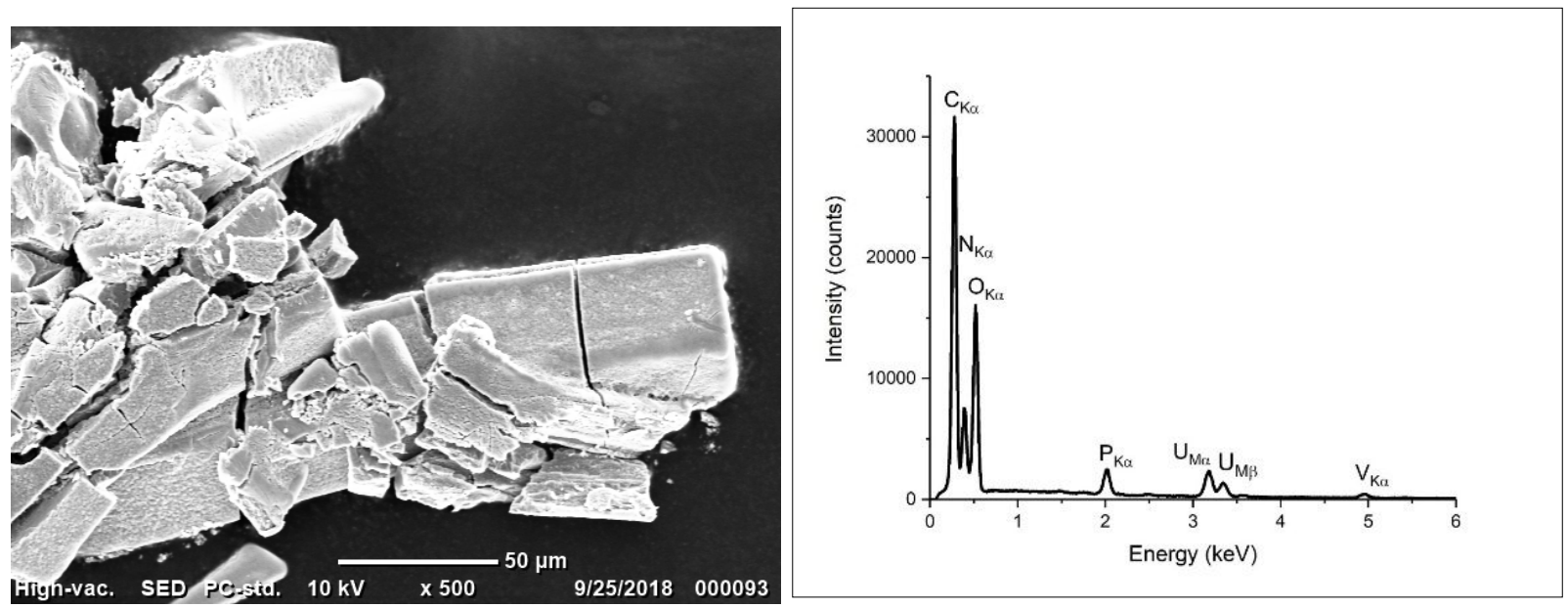
Figure S3. X-ray fluorescence (XRF) spectroscopy was performed on crystals of $\left\{\boldsymbol{U}_{\boldsymbol{\delta}} \boldsymbol{V}_{\boldsymbol{b}}\right\}^{-}$ monomer, $\left\{U_{8} V_{6}\right\}$-dimer, $\left\{U_{8} V_{6}\right\}$-chain, and $\left\{U_{6} V_{3}\right\}$ mounted on double-sided carbon tape.

\section{$\left\{U_{8} V_{6}\right\}$-monomer}
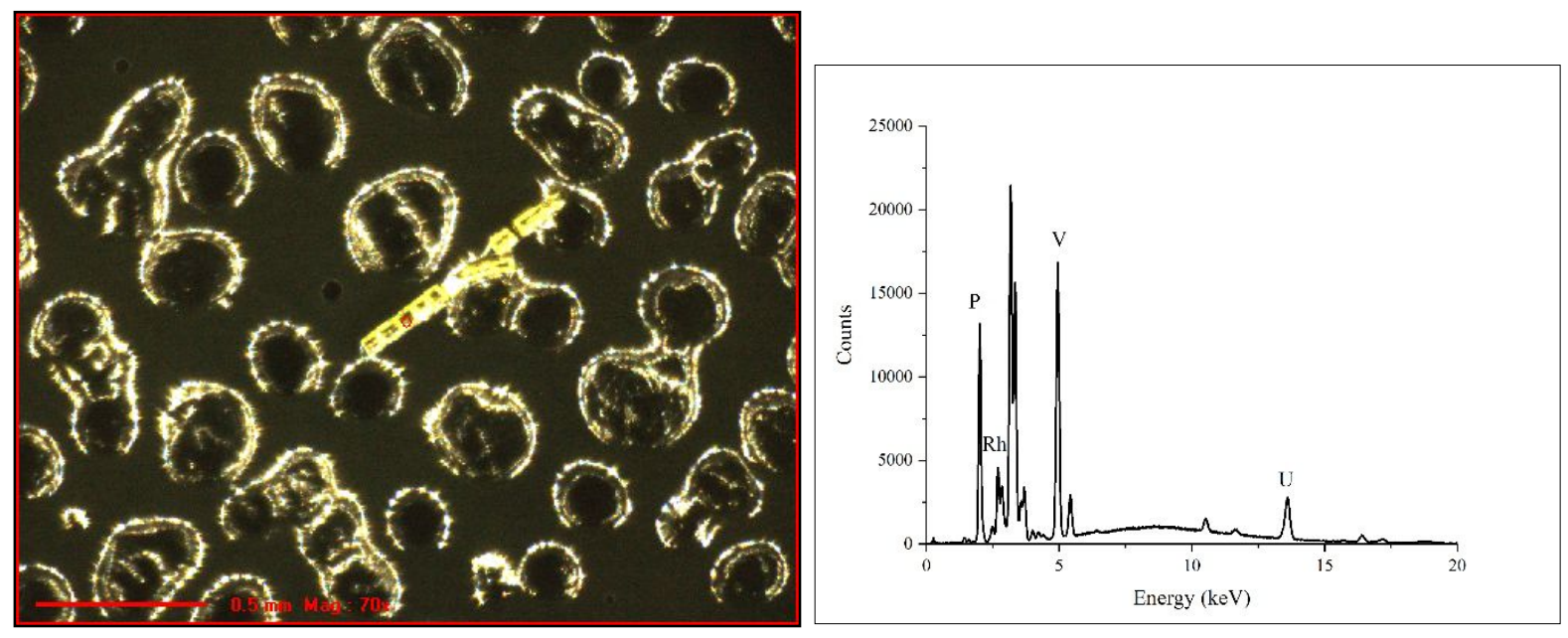

$\left\{U_{8} V_{6}\right\}$-dimer
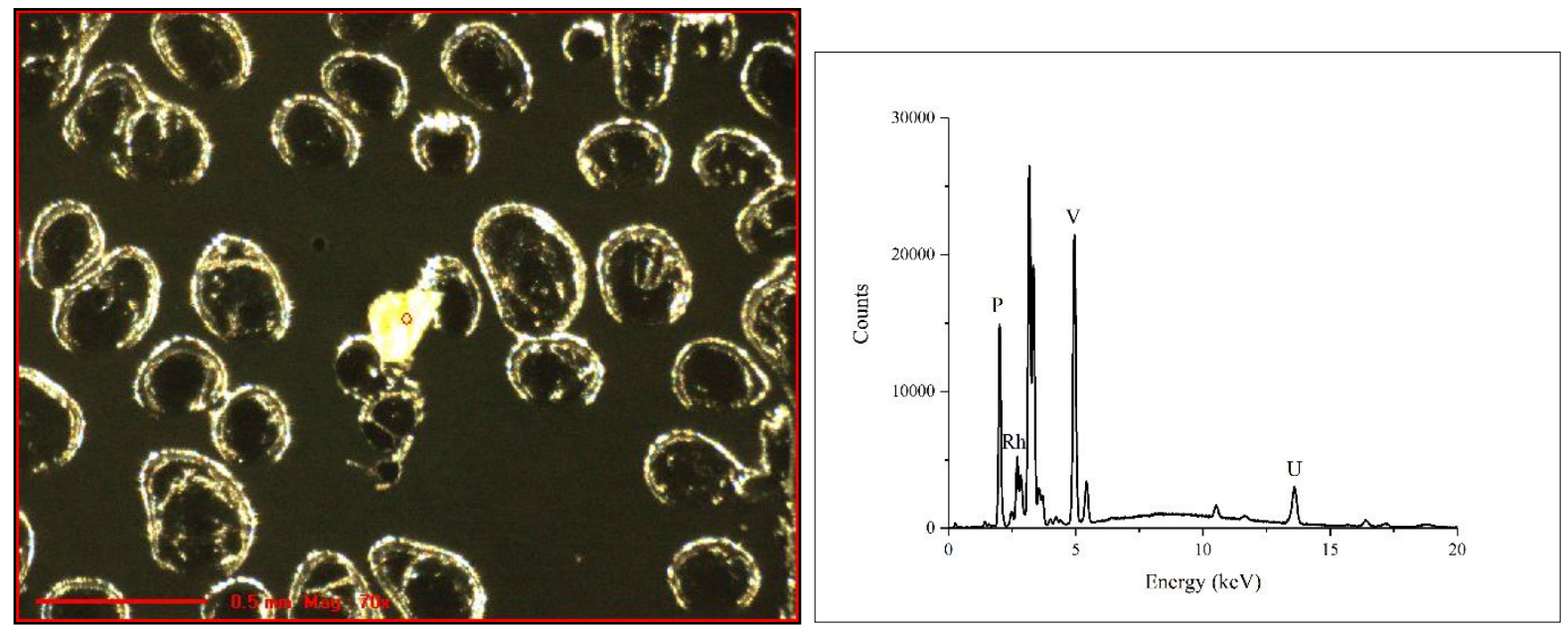
$\left\{\boldsymbol{U}_{8} \boldsymbol{V}_{6}\right\}$-chain - Sodium was not detected with the point analysis, so a map was obtained and measured for uranium (blue), vanadium (yellow), phosphorus (green), and sodium (red). Below the point analysis EDS spectrum are the maps for each of the elements.
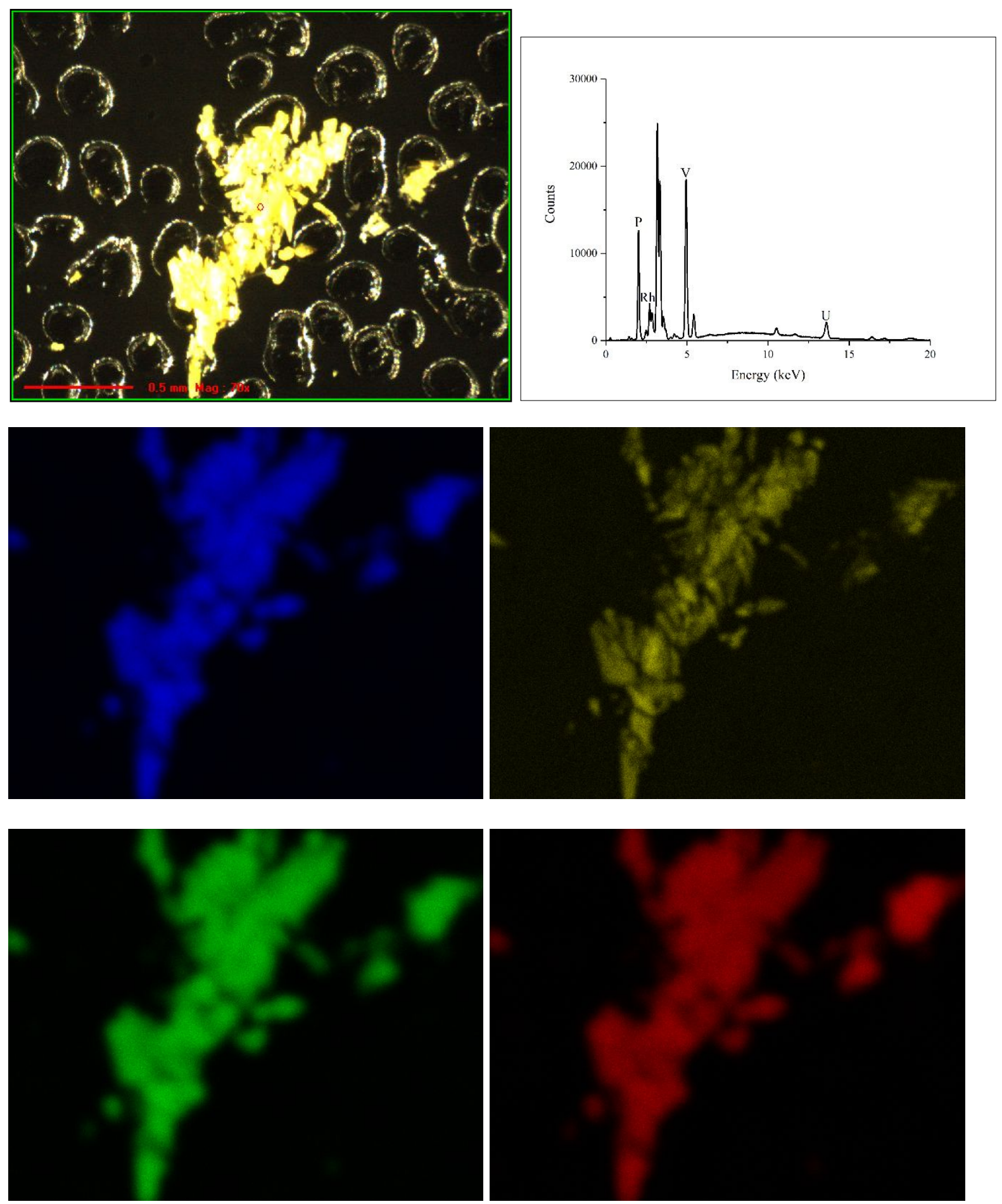
$\left\{U_{6} V_{3}\right\}$
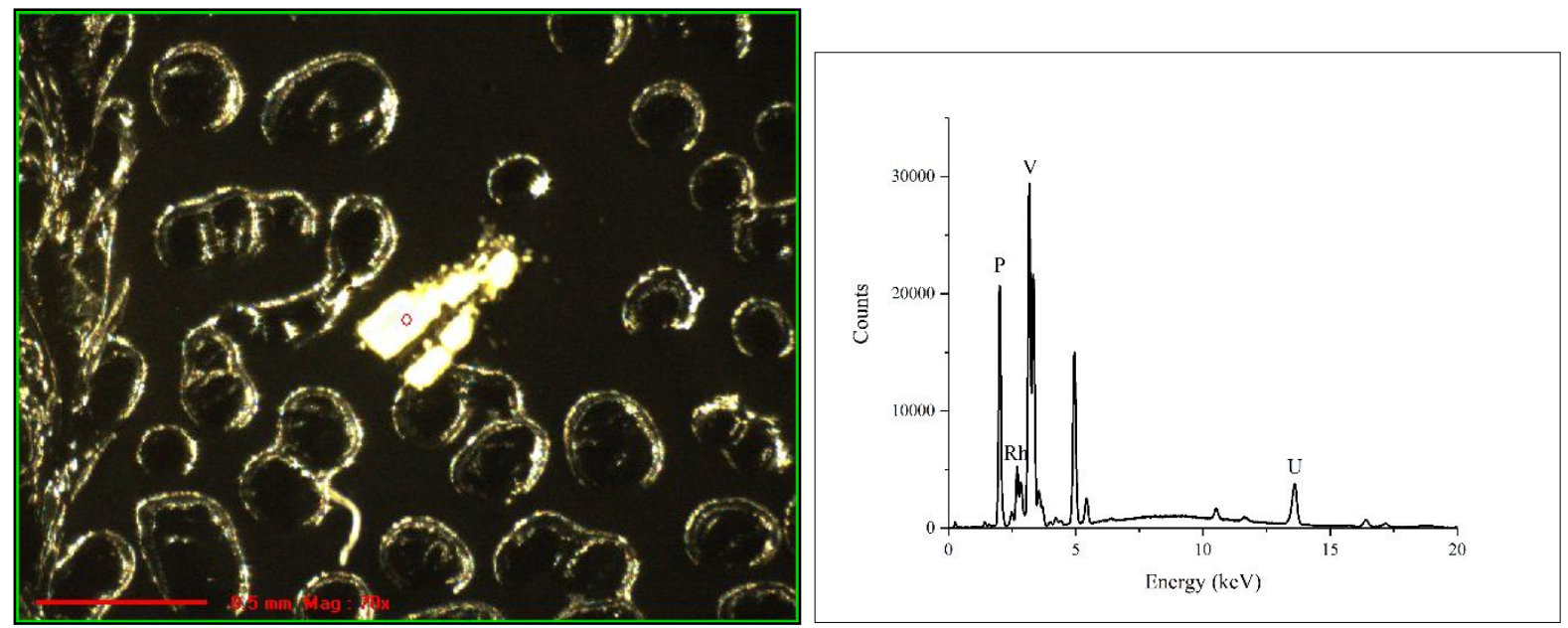
Figure S4. UV-Vis absorption spectra for $\left\{U_{8} V_{6}\right\}$-monomer, $\left\{U_{8} V_{6}\right\}$-dimer, $\left\{U_{8} V_{6}\right\}$-chain, and $\left\{\boldsymbol{U}_{6} \boldsymbol{V}_{3}\right\}$

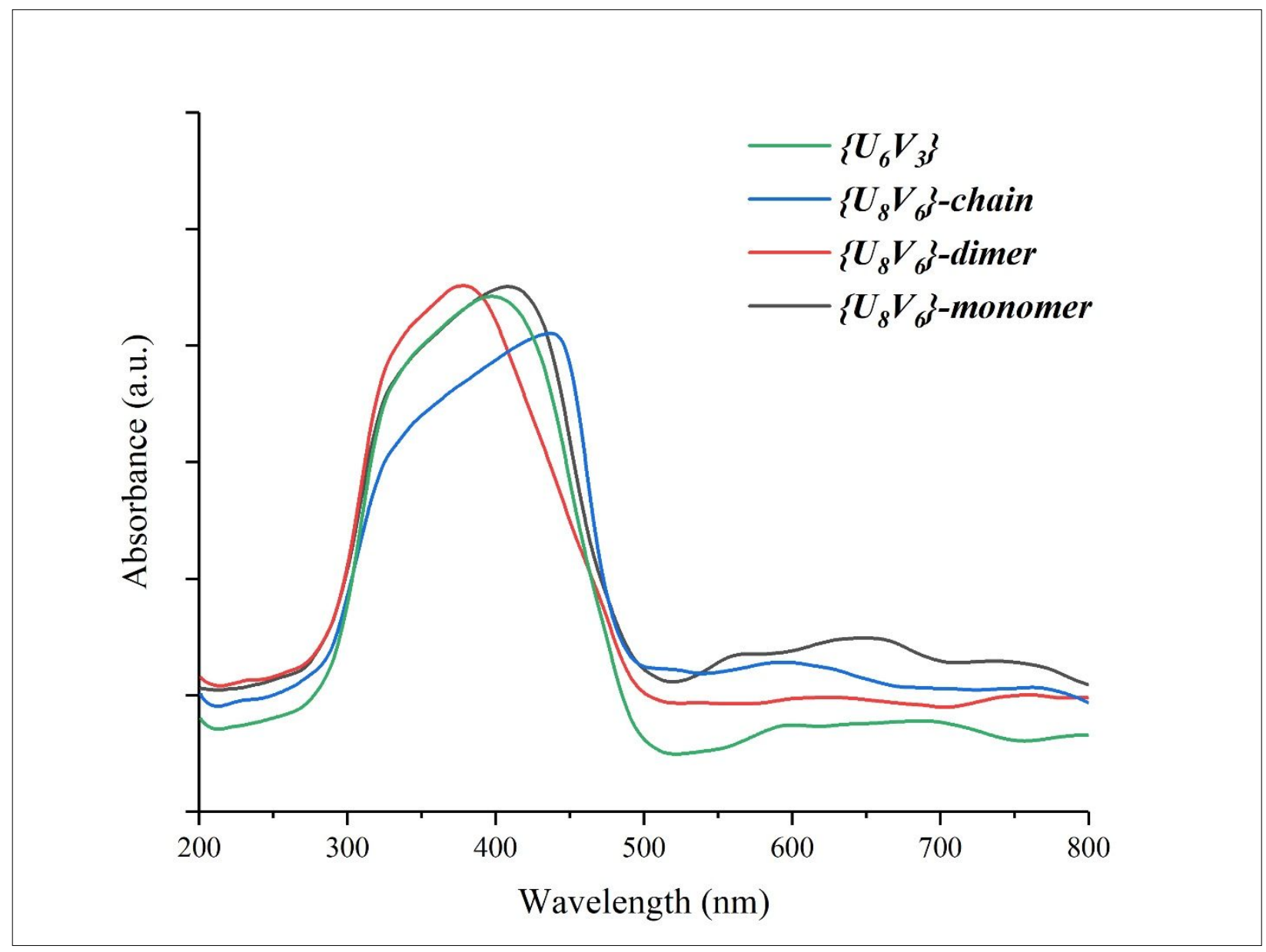


Figure S5. ESI mass spectra for methanol, ethanol, and acetonitrile solutions for initial (day 1) and aged (day 7) samples for $\left\{U_{8} V_{6}\right\}$-monomer, $\left\{U_{8} V_{6}\right\}$-dimer, $\left\{U_{8} V_{6}\right\}$-chain, and $\left\{U_{6} V_{3}\right\}$. The following is a summary of the abbreviations used in the peak assignment tables:

\begin{tabular}{|c|c|c|c|}
\hline \multicolumn{4}{|c|}{ Solvent } \\
\hline $\mathrm{MeOH}$ & Methanol, $\mathrm{CH}_{3} \mathrm{OH}$ & $\mathrm{EtOH}$ & Ethanol, $\mathrm{CH}_{3} \mathrm{CH}_{2} \mathrm{OH}$ \\
\hline $\mathrm{MeCN}$ & Acetonitrile, $\mathrm{CH}_{3} \mathrm{CN}$ & $\mathrm{H}_{2} \mathrm{O}$ & Water, $\mathrm{H}_{2} \mathrm{O}$ \\
\hline \multicolumn{4}{|c|}{ Cations } \\
\hline $\mathrm{H}$ & Proton, $\mathrm{H}^{+}$ & EMIm & EMIm, $\mathrm{C}_{6} \mathrm{~N}_{2} \mathrm{H}_{11}{ }^{+}$ \\
\hline \multirow[t]{2}{*}{$\mathrm{U}$} & Uranyl ion, $\mathrm{UO}_{2}{ }^{2+}$ & & \\
\hline & \multicolumn{2}{|c|}{ Anions } & \\
\hline $\mathrm{V}_{6}$ & $\mathrm{~V}_{6} \mathrm{O}_{22}{ }^{14-}$ & $\mathrm{V}_{3}$ & $\mathrm{~V}_{3} \mathrm{O}_{12}{ }^{9-}$ \\
\hline $\mathrm{Vt}$ & Vanadate, $\mathrm{VO}_{4}{ }^{3-}$ & $\mathrm{Ph}$ & phosphate, $\mathrm{PO}_{4}^{3-}$ \\
\hline $\mathrm{P}$ & diethyl phosphate, & Et & Ethyl, $\mathrm{CH}_{3} \mathrm{CH}_{2}$ \\
\hline & {$\left[\mathrm{PO}_{4}\left(\mathrm{C}_{2} \mathrm{H}_{5}\right)_{2}\right]^{-}$} & & \\
\hline
\end{tabular}


Day 1 - Methanol

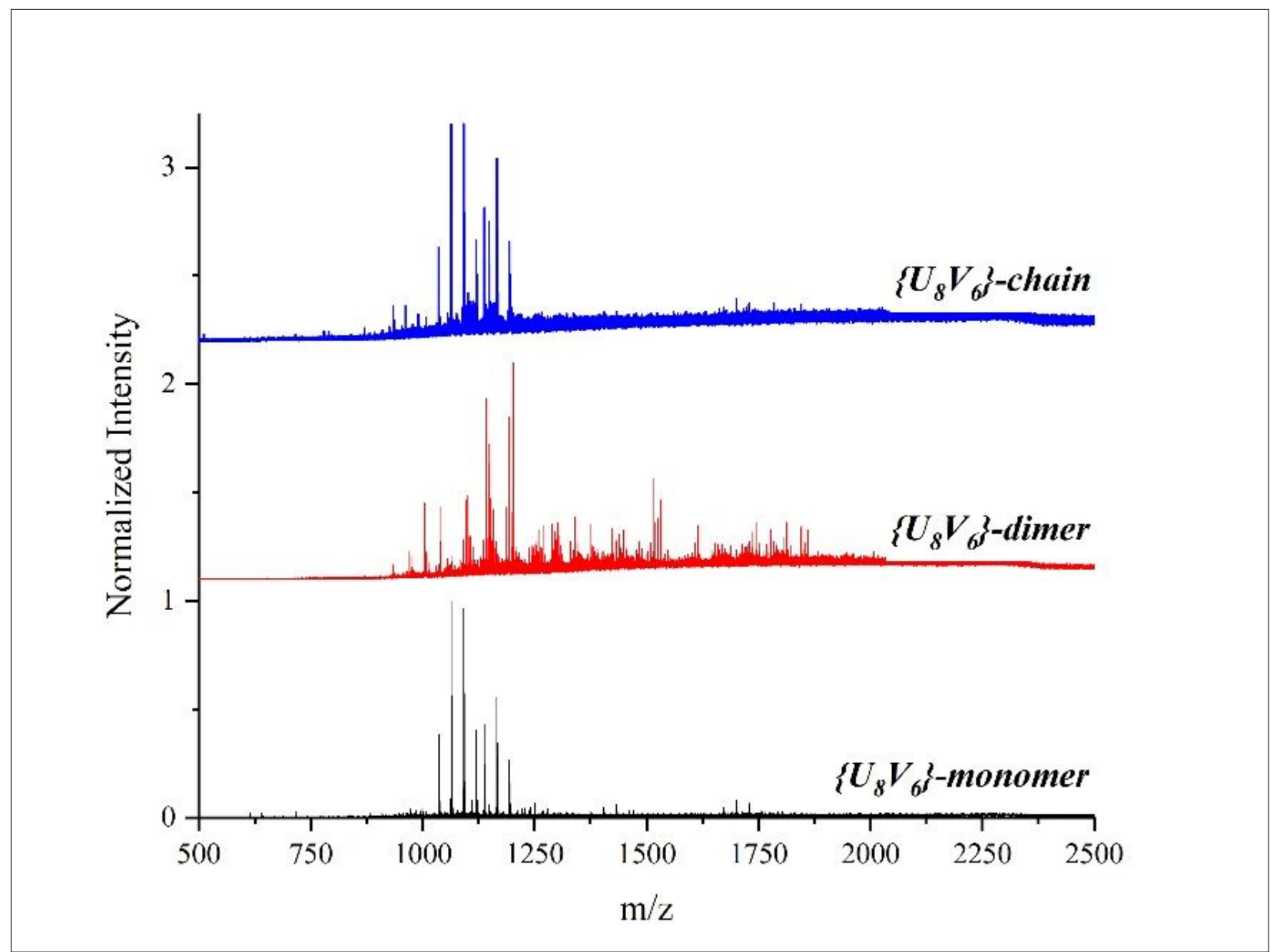

\begin{tabular}{|c|c|c|c|}
\hline Experimental $(\mathrm{m} / z)$ & Calculated $(\mathrm{m} / \mathrm{z})$ & Assignment & Materials \\
\hline 1035.8 & 1035.1 & $\mathrm{H}(\mathrm{EMIm})\left(\mathrm{U}_{2} \mathrm{Vt}_{2} \mathrm{P}\right)^{1-}$ & $\begin{array}{c}\left\{U_{8} V_{6}\right\} \text {-monomer } \\
\text {-chain }\end{array}$ \\
\hline 1063.8 & 1063.3 & $(\mathrm{EMIm})_{4} \mathrm{UPh}_{3}(\mathrm{MeOH})_{2}{ }^{1-}$ & $\begin{array}{c}\left\{U_{8} V_{6}\right\} \text {-monomer } \\
\text {-chain }\end{array}$ \\
\hline 1091.8 & 1092.0 & $(\mathrm{EMIm})\left(\mathrm{U}_{2} \mathrm{~V}_{3}\right)(\mathrm{MeOH})_{3}{ }^{1-}$ & $\begin{array}{c}\left\{U_{8} V_{6}\right\} \text {-monomer } \\
\text {-chain }\end{array}$ \\
\hline 1119.8 & 1120.0 & $\mathrm{H}_{2}\left(\mathrm{U}_{2} \mathrm{~V}_{3} \mathrm{P}\right)(\mathrm{Et})\left(\mathrm{H}_{2} \mathrm{O}\right)(\mathrm{MeOH})^{1-}$ & $\begin{array}{c}\left\{U_{8} V_{6}\right\} \text {-monomer } \\
\text {-chain }\end{array}$ \\
\hline
\end{tabular}




\begin{tabular}{|c|c|c|c|}
\hline 1137.8 & 1137.5 & $\mathrm{H}_{2}(\mathrm{EMIm})_{5}\left(\mathrm{VtP}_{2} \mathrm{Ph}\right)(\mathrm{MeOH})_{2}{ }^{1-}$ & $\begin{array}{c}\left\{U_{8} V_{6\}}\right\} \text {-monomer }, \\
\text {-chain }\end{array}$ \\
\hline 1165.8 & 1165.6 & $\mathrm{H}_{2}(\mathrm{EMIm})_{6}\left(\mathrm{VtPh}_{2}\right)(\mathrm{MeOH})_{6}{ }^{1-}$ & $\begin{array}{c}\left\{U_{8} V_{6\}}\right\} \text {-monomer }, \\
\text {-chain }\end{array}$ \\
\hline 1193.9 & 1193.5 & $(\mathrm{EMIm})_{5}\left(\mathrm{P}_{3} \mathrm{Vt}\right)(\mathrm{MeOH})_{2}{ }^{1-}$ & $\begin{array}{c}\left\{U_{8} V_{6\}}\right\} \text {-monomer }, \\
\text {-chain }\end{array}$ \\
\hline 1432.1 & 1433.7 & $(\mathrm{EMIm})_{7}\left(\mathrm{P}_{2} \mathrm{Ph}_{2}\right)(\mathrm{MeOH})_{5}{ }^{1-}$ & $\begin{array}{c}\left\{U_{8} V_{6\}}\right\} \text {-monomer }, \\
\text {-chain }\end{array}$ \\
\hline 1700.2 & 1701.7 & $\left(\mathrm{EMIm}_{9}\left(\mathrm{UVtPh}_{3}\right)(\mathrm{MeOH})^{1-}\right.$ & $\begin{array}{c}\left\{U_{8} V_{6\}}\right\} \text {-monomer }, \\
\text {-chain }\end{array}$ \\
\hline
\end{tabular}

\begin{tabular}{|c|c|c|c|}
\hline $\begin{array}{c}\text { Experimental } \\
(\boldsymbol{m} / \boldsymbol{z})\end{array}$ & $\begin{array}{c}\text { Calculated } \\
(\boldsymbol{m} / \boldsymbol{z})\end{array}$ & Assignment & Materials \\
\hline 1004.6 & 1004.6 & $\mathrm{H}(\mathrm{EMIm})_{4}\left[\mathrm{U}_{10}\left(\mathrm{~V}_{6}\right)_{2} \mathrm{P}_{2}\right](\mathrm{MeOH})_{8}{ }^{5-}$ & $\left\{U_{8} V_{6}\right\}$-dimer \\
\hline 1038.6 & 1038.2 & $\mathrm{H}(\mathrm{EMIm})_{5}\left[\mathrm{U}_{10}\left(\mathrm{~V}_{6}\right)_{2} \mathrm{P}_{3}\right](\mathrm{MeOH})_{5}{ }^{5-}$ & $\left\{U_{8} V_{6}\right\}$-dimer \\
\hline 1096.4 & 1095.8 & $(\mathrm{EMIm})_{3}\left(\mathrm{U}_{6} \mathrm{~V}_{6} \mathrm{P}_{4}\right)(\mathrm{MeOH})_{2}{ }^{3-}$ & $\left\{U_{8} V_{6}\right\}$-dimer \\
\hline 1141.7 & 1141.7 & $\mathrm{HEMIm}\left(\mathrm{U}_{7} \mathrm{~V}_{6} \mathrm{P}_{5}\right)^{3-}$ & $\left\{U_{8} V_{6}\right\}$-dimer \\
\hline 1202.7 & 1202.5 & $\mathrm{H}(\mathrm{EMIm})_{6}\left[\mathrm{U}_{6}\left(\mathrm{~V}_{3}\right)_{2} \mathrm{P}_{4}\right]\left(\mathrm{H}_{2} \mathrm{O}\right)^{3-}$ & $\left\{U_{8} V_{6}\right\}$-dimer \\
\hline 1517.6 & 1518.1 & $(\mathrm{EMIm})_{5}\left[\mathrm{U}_{6}\left(\mathrm{~V}_{3}\right)_{2} \mathrm{P}\right]^{2-}$ & $\left\{U_{8} V_{6}\right\}$-dimer \\
\hline 1845.2 & 1845.5 & $(\mathrm{EMIm})_{8}\left(\mathrm{U}_{4} \mathrm{~V}_{3} \mathrm{P}_{9}\right)^{2-}$ & $\left\{U_{8} V_{6}\right\}$-dimer \\
\hline
\end{tabular}




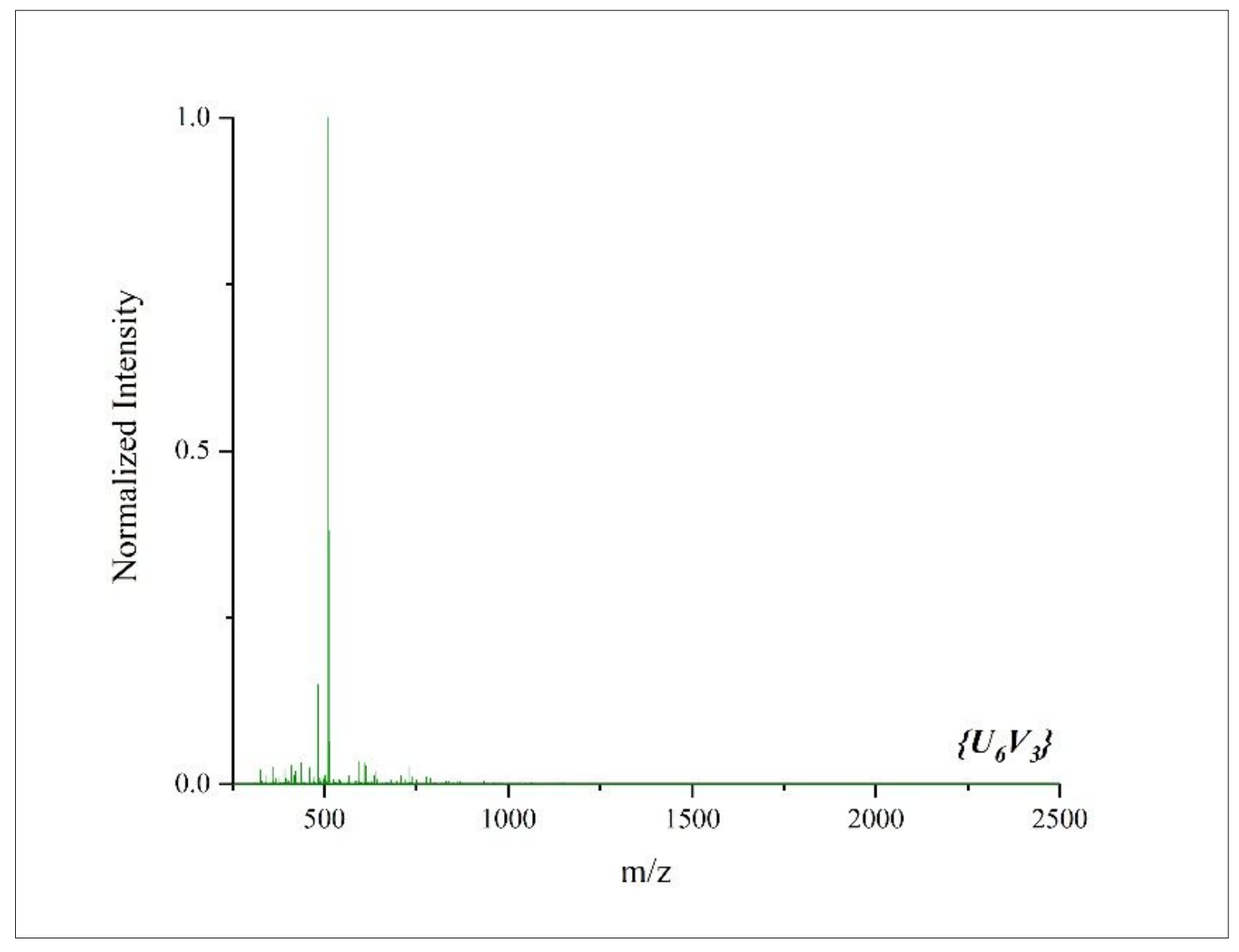

\begin{tabular}{|c|c|c|c|}
\hline Experimental $(\boldsymbol{m} / \mathbf{z})$ & Calculated & Assignment & Materials \\
\hline & $(\boldsymbol{m} / \mathbf{z})$ & \\
\hline 483.3 & 483.0 & $\mathrm{H}_{3} \mathrm{UPhVt}^{1-}$ & $\left\{U_{6} V_{3}\right\}$ \\
\hline 511.3 & 511.0 & $\mathrm{H}_{2} \mathrm{UPhVt}(\mathrm{Et})^{1-}$ & $\left\{U_{6} V_{3}\right\}$ \\
\hline
\end{tabular}


Day 7 - Methanol

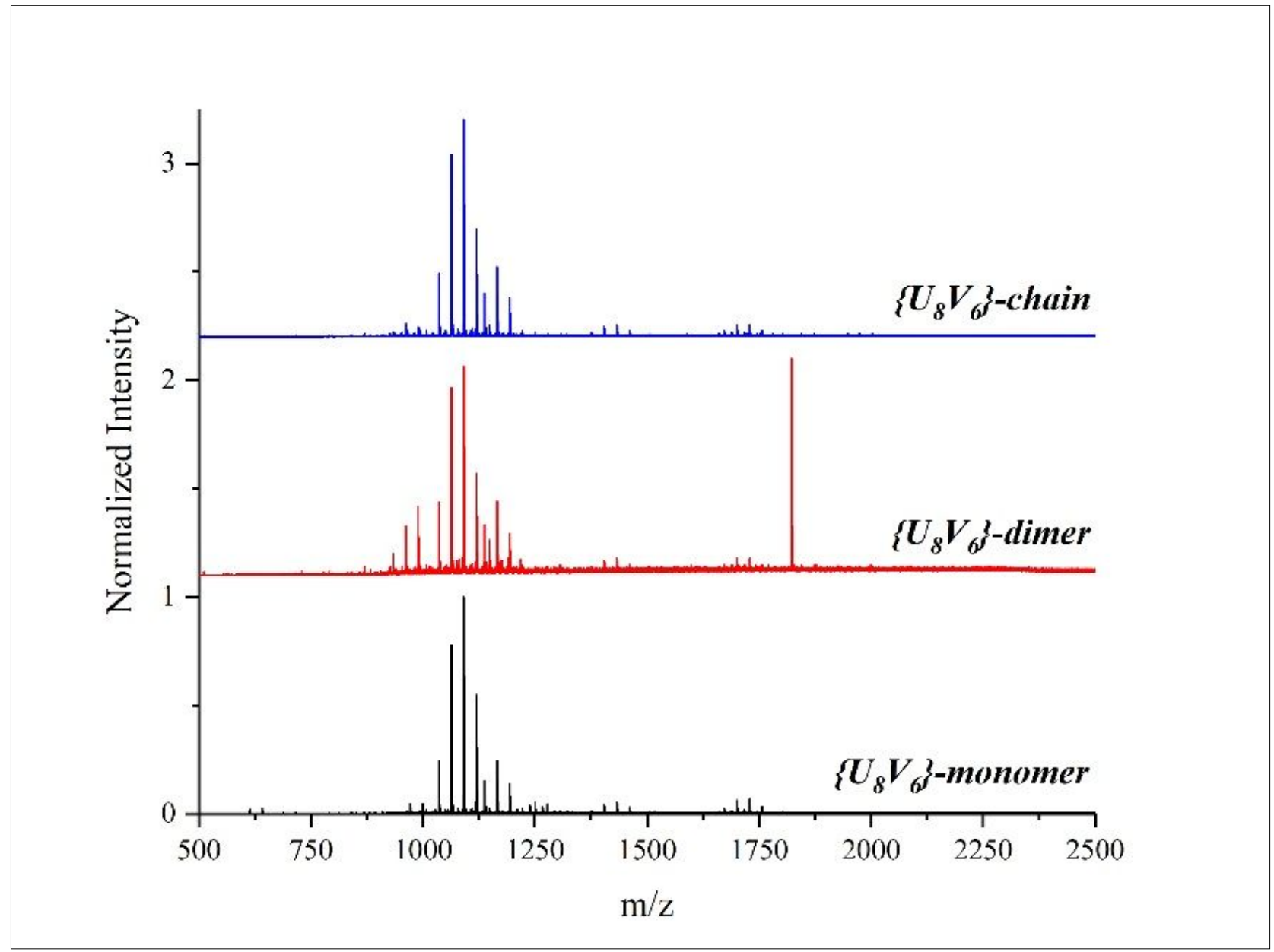

\begin{tabular}{|c|c|c|c|}
\hline $\begin{array}{c}\text { Experimental } \\
(m / z)\end{array}$ & $\begin{array}{c}\text { Calculated } \\
\qquad(m / z)\end{array}$ & Assignment & Materials \\
\hline 961.6 & 961.0 & $\mathrm{H}_{8}\left(\mathrm{UV}_{3} \mathrm{P}_{2}\right)(\mathrm{MeOH})^{1-}$ & $\left\{U_{8} V_{6}\right\}$-dimer \\
\hline 988.1 & 988.2 & $\mathrm{H}_{3}(\mathrm{EMIm})_{4}\left(\mathrm{U}_{2} \mathrm{~V}_{3} \mathrm{P}_{4}\right)(\mathrm{MeOH})^{2-}$ & $\left\{U_{8} V_{6}\right\}$-dimer \\
\hline 1035.8 & 1035.4 & $\mathrm{H}(\mathrm{EMIm})_{5}\left(\mathrm{PVt}_{2}\right)(\mathrm{MeOH})_{3}{ }^{1-}$ & $\begin{array}{l}\left\{U_{8} V_{6}\right\} \text {-monomer } \\
\text {-dimer, -chain }\end{array}$ \\
\hline 1063.8 & 1063.3 & $\left(\mathrm{EMIm}_{4}\left(\mathrm{UPh}_{3}\right)(\mathrm{MeOH})_{2}{ }^{1-}\right.$ & $\begin{array}{l}\left\{U_{8} V_{6}\right\} \text {-monomer } \\
\text {-dimer, -chain }\end{array}$ \\
\hline
\end{tabular}




\begin{tabular}{|c|c|c|c|}
\hline 1091.8 & 1092.0 & $(\mathrm{EMIm})\left(\mathrm{U}_{2} \mathrm{~V}_{3}\right)(\mathrm{MeOH})_{3}{ }^{1-}$ & $\begin{array}{c}\left\{U_{8} V_{6}\right\} \text {-monomer } \\
\text {-dimer, -chain }\end{array}$ \\
\hline 1119.8 & 1120.0 & $\mathrm{H}_{2}\left(\mathrm{U}_{2} \mathrm{~V}_{3} \mathrm{P}\right)(\mathrm{Et})\left(\mathrm{H}_{2} \mathrm{O}\right)(\mathrm{MeOH})^{1}$ & $\begin{array}{c}\left\{U_{8} V_{6}\right\} \text {-monomer } \\
\text {-dimer, -chain }\end{array}$ \\
\hline 1165.8 & 1165.5 & $\mathrm{H}_{2}(\mathrm{EMIm})_{3}\left(\mathrm{P}_{3} \mathrm{Vt}\right)(\mathrm{MeOH})_{8}{ }^{1-}$ & $\begin{array}{c}\left\{U_{8} V_{6}\right\} \text {-monomer }, \\
\text {-dimer, -chain }\end{array}$ \\
\hline 1432.1 & 1431.6 & $\mathrm{H}(\mathrm{EMIm})_{8}\left(\mathrm{PPhVt}_{2}\right)(\mathrm{MeOH})_{2}{ }^{1-}$ & $\begin{array}{c}\left\{U_{8} V_{6}\right\}-\text { monomer } \\
\text {-dimer, -chain }\end{array}$ \\
\hline 1728.1 & 1727.8 & $\mathrm{H}(\mathrm{EMIm})_{9}\left(\mathrm{P}_{2} \mathrm{PhVt}_{2}\right)(\mathrm{MeOH})_{3}{ }^{1-}$ & $\begin{array}{c}\left\{U_{8} V_{6}\right\}-\text { monomer } \\
\text {-dimer, -chain }\end{array}$ \\
\hline 1823.2 & 1823.4 & $\mathrm{H}_{3}(\mathrm{EMIm})_{4}\left(\mathrm{U}_{2} \mathrm{~V}_{3} \mathrm{P}_{3}\right)(\mathrm{MeOH})^{1-}$ & $\left\{U_{8} V_{6}\right\}$-dimer \\
\hline
\end{tabular}

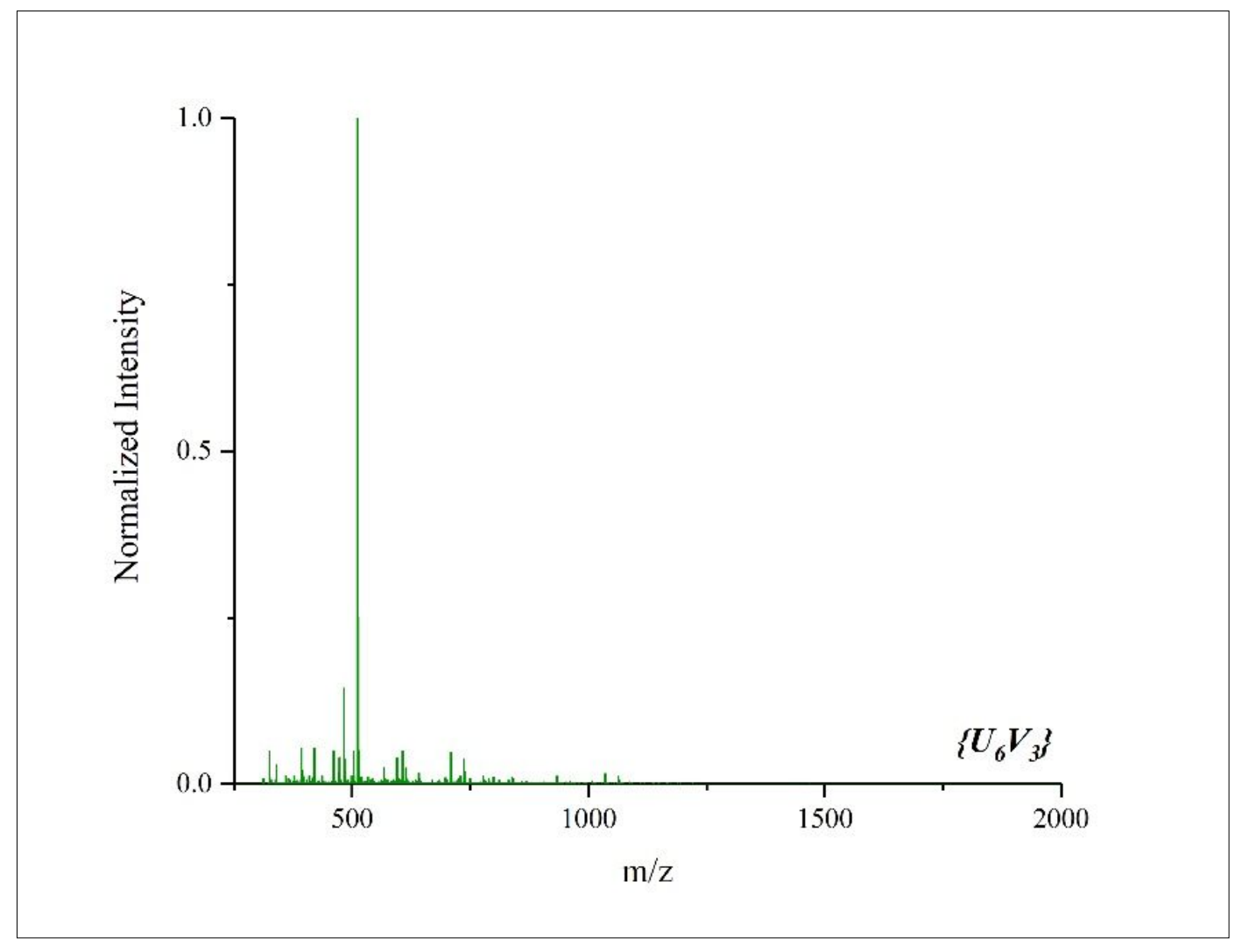




\begin{tabular}{|c|c|c|c|}
\hline Experimental $(\mathbf{m} / \mathbf{z})$ & Calculated & Assignment & Materials \\
\hline & $(\boldsymbol{m} / \mathbf{z})$ & & \\
\hline 483.3 & 483.0 & $\mathrm{H}_{3} \mathrm{UPhVt}^{1-}$ & $\left\{U_{6} V_{3}\right\}$ \\
\hline 511.3 & 511.0 & $\mathrm{H}_{2} \mathrm{UPhVt}(\mathrm{Et})^{1-}$ & $\left\{U_{6} V_{3}\right\}$ \\
\hline
\end{tabular}

Day 1 - Ethanol

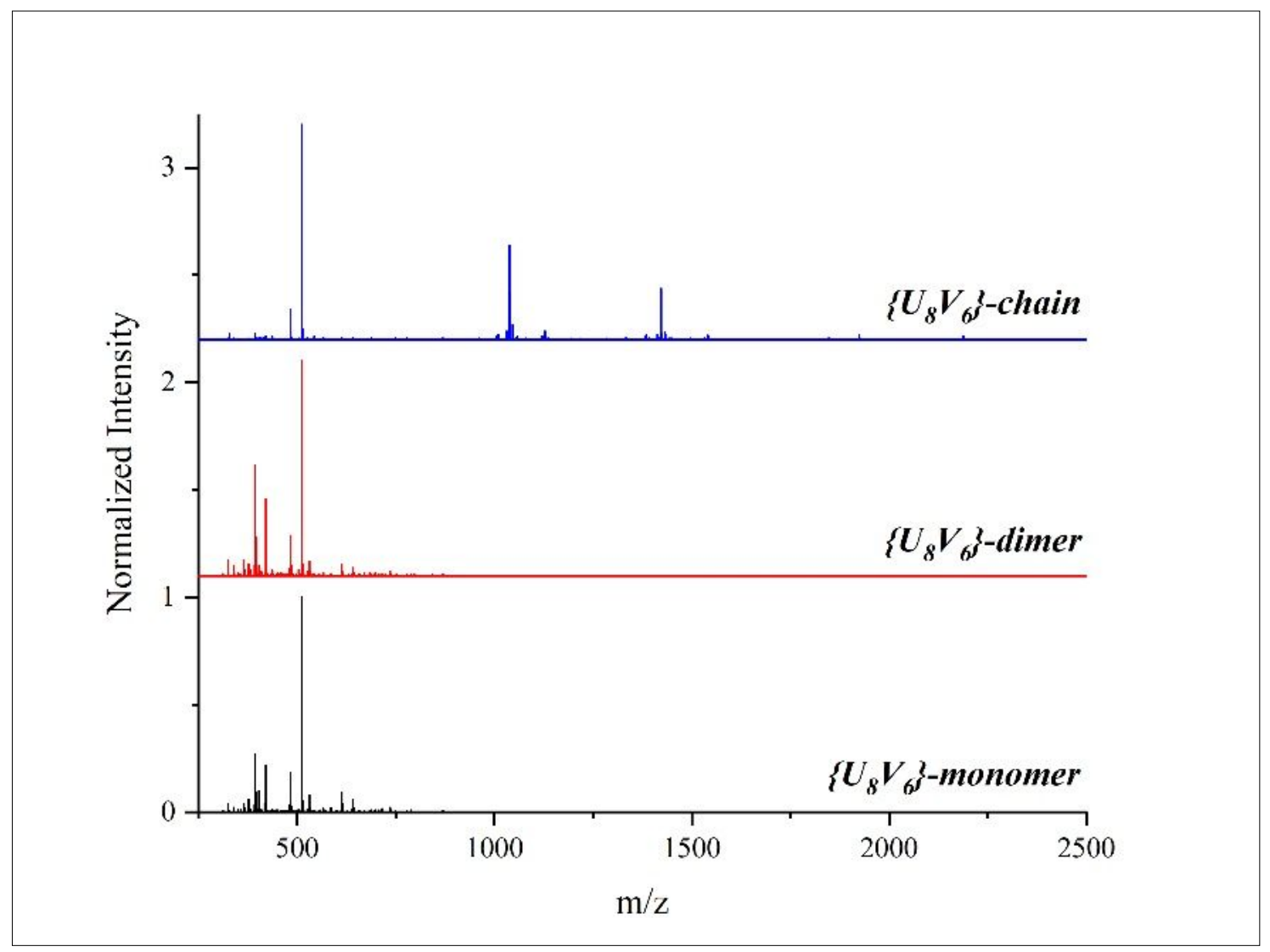

\begin{tabular}{|c|c|c|c|}
\hline Experimental $(\mathbf{m} / \mathbf{z})$ & Calculated $(\mathbf{m} / \mathbf{z})$ & Assignment & Materials \\
\hline 393.3 & 393.2 & $\mathrm{H}_{2} \mathrm{Vt}(\mathrm{EtOH})_{6}{ }^{1-}$ & $\left\{U_{8} V_{6}\right\}$-monomer, \\
& & & -dimer, -chain \\
\hline
\end{tabular}




\begin{tabular}{|c|c|c|c|}
\hline 420.3 & 419.3 & $\mathrm{H}_{2} \mathrm{Ph}(\mathrm{EtOH})_{7}^{1-}$ & $\begin{array}{l}\left\{U_{8} V_{6\}}\right\} \text {-monomer } \\
\text {-dimer, -chain }\end{array}$ \\
\hline 483.3 & 483.0 & $\mathrm{H}_{3} \mathrm{UPhVt}^{1-}$ & $\begin{array}{l}\left\{U_{8} V_{6}\right\} \text {-monomer } \\
\text {-dimer, -chain }\end{array}$ \\
\hline 511.4 & 511.0 & $\mathrm{H}_{2} \mathrm{UPhVt}(\mathrm{Et})^{1-}$ & $\begin{array}{c}\left\{U_{8} V_{6}\right\} \text {-monomer } \\
\text {-dimer, -chain }\end{array}$ \\
\hline 613.6 & 613.0 & $\mathrm{H}_{2}(\mathrm{EMIm}) \mathrm{UVt}_{2}{ }^{1-}$ & $\begin{array}{l}\left\{U_{8} V_{6\}}\right\} \text {-monomer } \\
\text {-dimer, -chain }\end{array}$ \\
\hline 641.6 & 641.0 & $\operatorname{HEMIm}\left(\mathrm{UVt}_{2}\right)(\mathrm{Et})^{1-}$ & $\begin{array}{l}\left\{U_{8} V_{6\}}\right\} \text {-monomer } \\
\text {-dimer, -chain }\end{array}$ \\
\hline 1038.6 & 1038.6 & $\mathrm{H}(\mathrm{EMIm})\left(\mathrm{U}_{8} \mathrm{~V}_{6} \mathrm{P}_{8}\right)^{4-}$ & $\left\{U_{8} V_{6}\right\}$-chain \\
\hline 1421.8 & 1421.8 & $\mathrm{H}(\mathrm{EMIm})_{2}\left(\mathrm{U}_{8} \mathrm{~V}_{6} \mathrm{P}_{8}\right)^{3-}$ & $\left\{U_{8} V_{6}\right\}$-chain \\
\hline 1921.1 & 1920.2 & $\mathrm{H}_{5}(\mathrm{EMIm})_{3}\left(\mathrm{U}_{6} \mathrm{~V}_{6} \mathrm{P}_{8}\right)^{2-}$ & $\left\{U_{8} V_{6}\right\}$-chain \\
\hline 2188.2 & 2188.2 & $\mathrm{H}(\mathrm{EMIm})_{3}\left(\mathrm{U}_{8} \mathrm{~V}_{6} \mathrm{P}_{8}\right)^{2-}$ & $\left\{U_{8} V_{6}\right\}$-chain \\
\hline
\end{tabular}




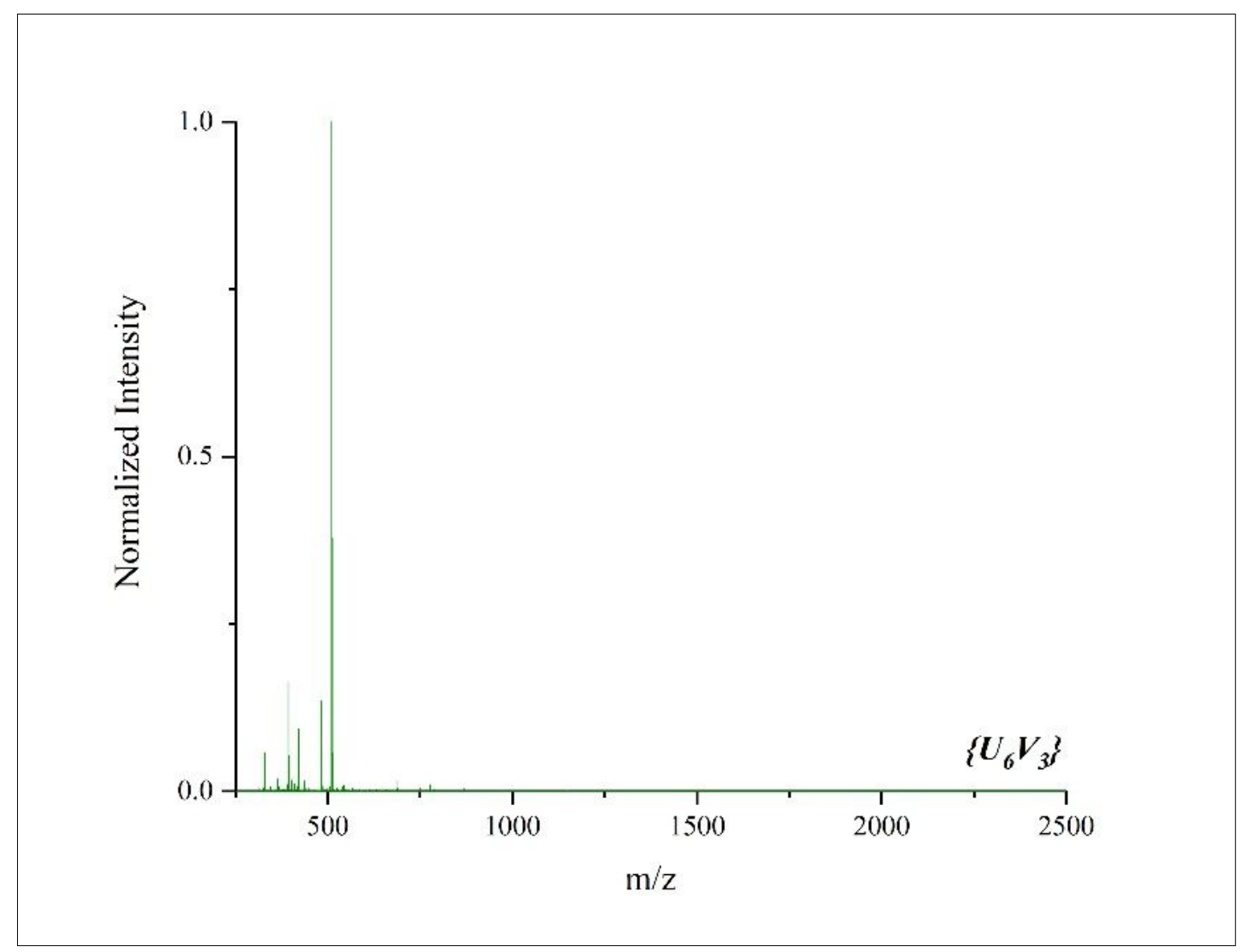

\begin{tabular}{|c|c|c|c|}
\hline Experimental $(\boldsymbol{m} / \mathbf{z})$ & Calculated $(\boldsymbol{m} / \mathbf{z})$ & Assignment & Materials \\
\hline 393.3 & 393.2 & $\mathrm{H}_{2} \mathrm{Vt}(\mathrm{EtOH})_{6}{ }^{1-}$ & $\left\{U_{6} V_{3}\right\}$ \\
\hline 420.3 & 419.3 & $\mathrm{H}_{2} \mathrm{Ph}(\mathrm{EtOH})_{7}{ }^{1-}$ & $\left\{U_{6} V_{3}\right\}$ \\
\hline 483.3 & 483.0 & $\mathrm{H}_{3} \mathrm{UVtPh}^{1-}$ & $\left\{U_{6} V_{3}\right\}$ \\
\hline 511.3 & 511.0 & $\mathrm{H}_{2}(\mathrm{UVtPh})(\mathrm{Et})^{1-}$ & $\left\{U_{6} V_{3}\right\}$ \\
\hline
\end{tabular}


Day 7 - Ethanol

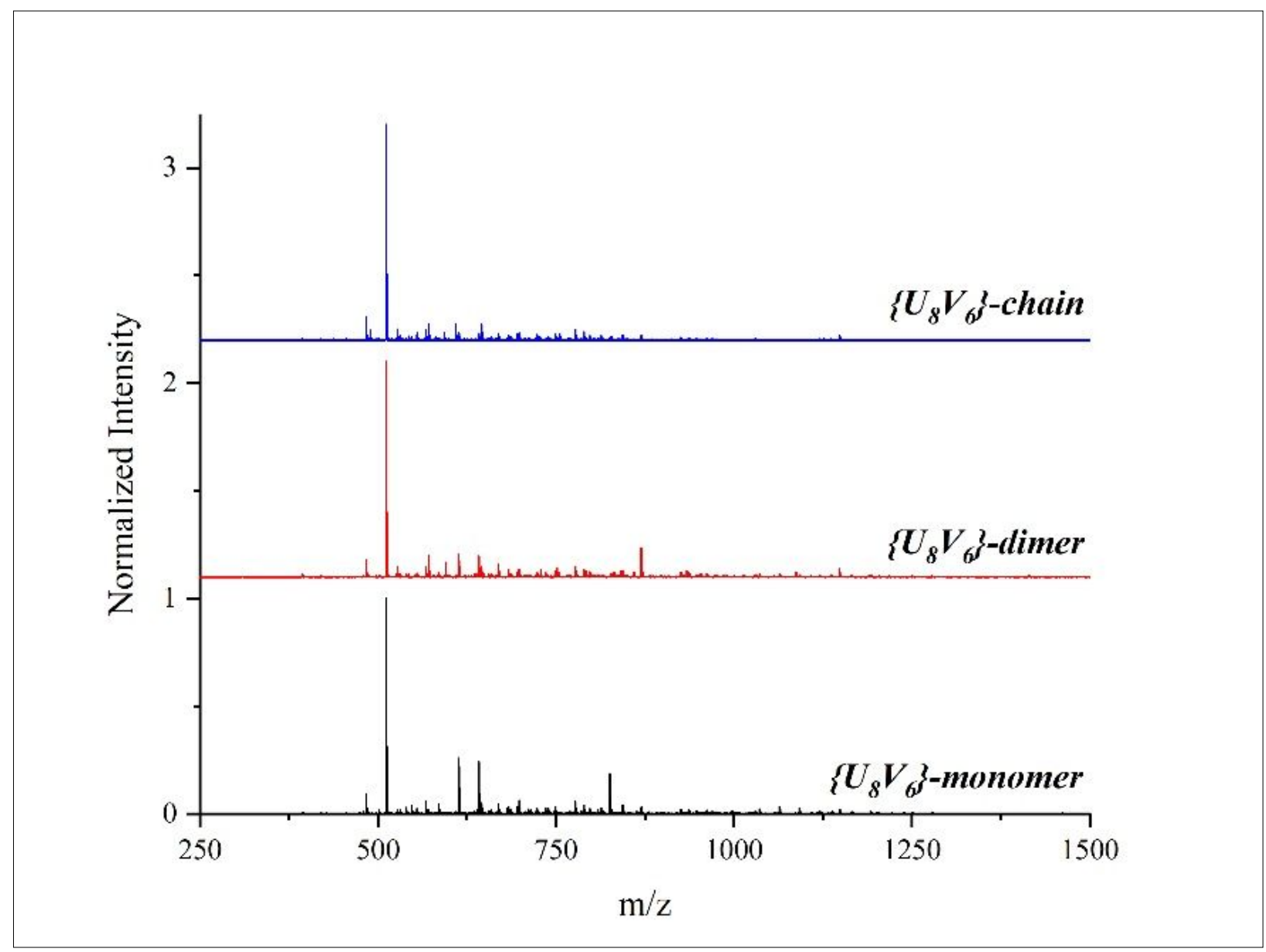

\begin{tabular}{|c|c|c|c|}
\hline Experimental $(\mathbf{m} / \mathbf{z})$ & Calculated $(\mathbf{m} / \mathbf{z})$ & Assignment & Materials \\
\hline 483.3 & 483.0 & $\mathrm{H}_{3} \mathrm{UVtPh}^{1-}$ & $\left\{U_{8} V_{6}\right\}_{\text {-monomer }}$ \\
& & & -dimer, -chain \\
\hline 511.4 & 511.0 & $\mathrm{H}_{2}(\mathrm{UVtPh})(\mathrm{Et})^{1-}$ & $\left\{U_{8} V_{6}\right\}$-monomer, \\
& & & - dimer, -chain \\
\hline 613.6 & 613.0 & $\mathrm{H}_{2}(\mathrm{EMIm}) \mathrm{UVt}_{2}{ }^{1-}$ & $\left\{U_{8} V_{6}\right\}$-monomer, \\
& & & - dimer, -chain \\
\hline
\end{tabular}




\begin{tabular}{|c|c|c|c|}
\hline 641.6 & 641.0 & $\operatorname{HEMIm}\left(\mathrm{UVt}_{2}\right)(\mathrm{Et})^{1-}$ & $\left\{U_{8} V_{6}\right\}$-monomer \\
& & & -dimer, -chain \\
\hline 825.3 & 825.3 & $\mathrm{H}_{4}(\mathrm{EMIm})_{4}(\mathrm{Ph})_{3}(\mathrm{EtOH})_{2}{ }^{1-}$ & $\left\{U_{8} V_{6}\right\}$-monomer \\
\hline 869.7 & 869.1 & $\mathrm{H}_{3}(\mathrm{EMIm})_{2}(\mathrm{UVt})(\mathrm{Et})^{1-}$ & $\left\{U_{8} V_{6}\right\}$-dimer \\
\hline
\end{tabular}

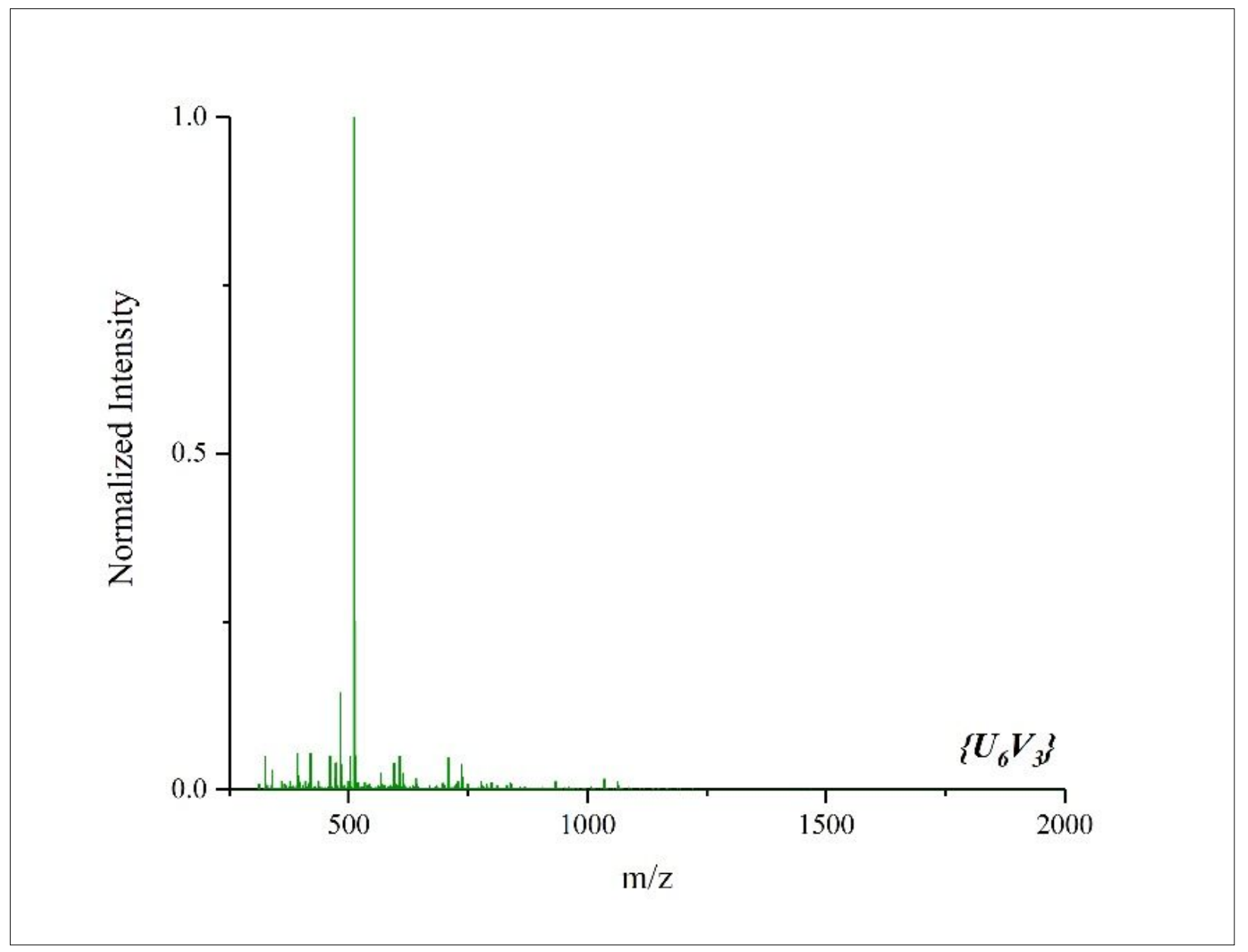

\begin{tabular}{|c|c|c|c|}
\hline Experimental $(\mathbf{m} / \mathbf{z})$ & Calculated $(\boldsymbol{m} / \mathbf{z})$ & Assignment & Materials \\
\hline 329.0 & 327.2 & $\mathrm{H}_{2}(\mathrm{Ph})(\mathrm{EtOH})_{5}{ }^{1-}$ & $\left\{U_{6} V_{3}\right\}$ \\
\hline 393.3 & 393.2 & $\mathrm{H}_{2} \mathrm{Vt}(\mathrm{EtOH})_{6}{ }^{1-}$ & $\left\{U_{6} V_{3}\right\}$ \\
\hline 420.3 & 421.0 & $\mathrm{UVt}\left(\mathrm{H}_{2} \mathrm{O}\right)_{2}{ }^{1-}$ & $\left\{U_{6} V_{3}\right\}$ \\
\hline
\end{tabular}




\begin{tabular}{|l|c|c|c|}
\hline 483.3 & 483.0 & $\mathrm{H}_{3} \mathrm{UVtPh}^{1-}$ & $\left\{U_{6} V_{3}\right\}$ \\
\hline 511.3 & 511.0 & $\mathrm{H}_{2}(\mathrm{UVtPh})(\mathrm{Et})^{1-}$ & $\left\{U_{6} V_{3}\right\}$ \\
\hline
\end{tabular}

Day 1 - Acetonitrile

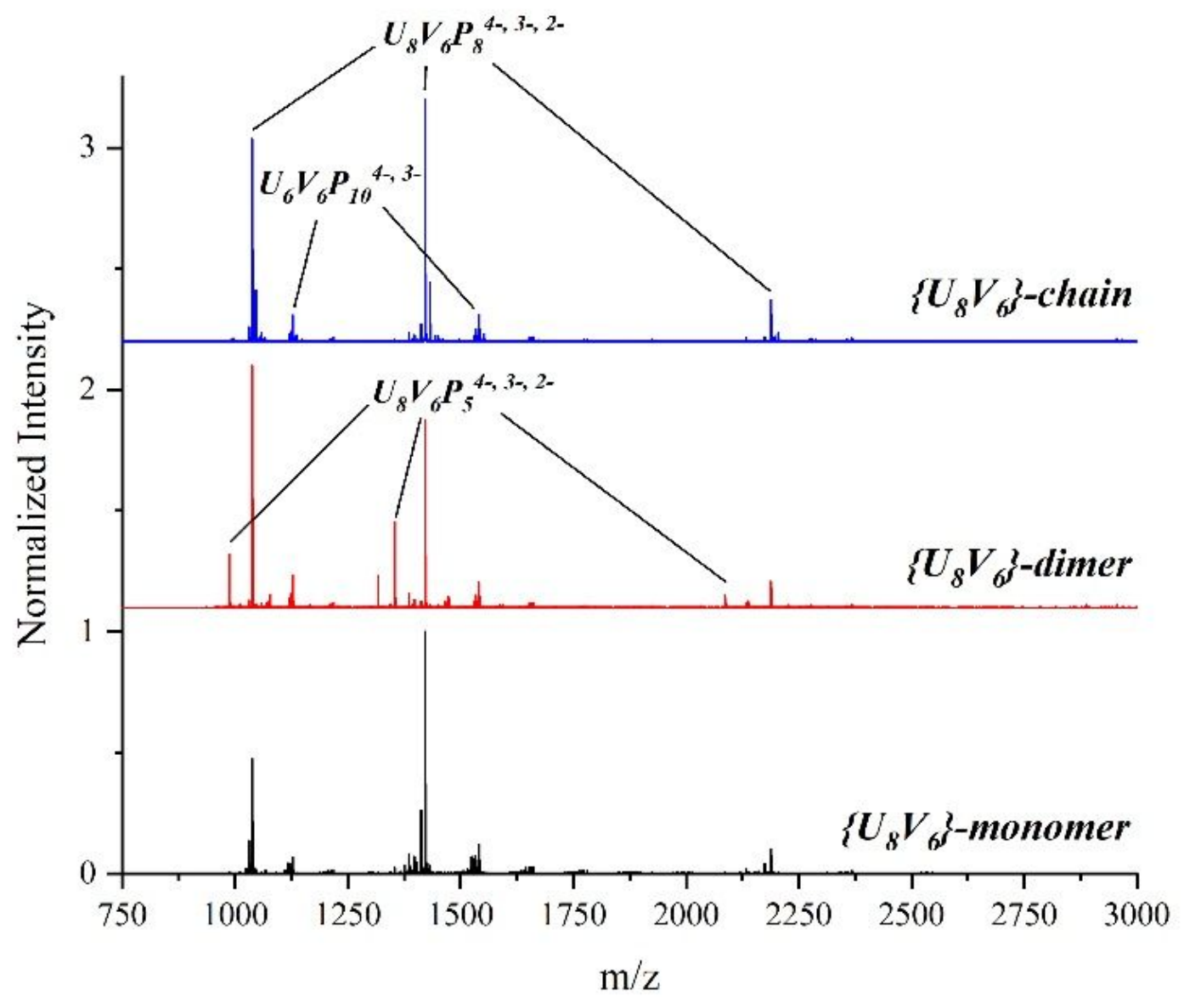

\begin{tabular}{|c|c|c|c|}
\hline Experimental & Calculated & Assignment & Materials \\
\hline$(\boldsymbol{m} / \mathbf{z})$ & $(\boldsymbol{m} / \mathbf{z})$ & & \\
\hline 987.8 & 987.8 & $\mathrm{H}_{2}(\mathrm{EMIm})\left[\mathrm{U}_{8}\left(\mathrm{~V}_{3}\right)_{2} \mathrm{P}_{5}\right](\mathrm{MeCN})_{5}\left(\mathrm{H}_{2} \mathrm{O}\right)^{4-}$ & $\left\{U_{8} V_{6}\right\}$-dimer \\
\hline 1038.6 & 1038.6 & $\mathrm{H}(\mathrm{EMIm})\left(\mathrm{U}_{8} \mathrm{~V}_{6} \mathrm{P}_{8}\right)^{4-}$ & $\left\{U_{8} V_{6}\right\}$-monomer \\
& & & - dimer, -chain \\
\hline
\end{tabular}




\begin{tabular}{|c|c|c|c|}
\hline 1128.2 & 1128.2 & $\mathrm{H}_{2}(\mathrm{EMIm})_{6}\left(\mathrm{U}_{6} \mathrm{~V}_{6} \mathrm{P}_{10}\right)\left(\mathrm{H}_{2} \mathrm{O}\right)_{2}^{4-}$ & $\begin{array}{l}\left\{U_{8} V_{6}\right\} \text {-monomer } \\
\text {-dimer, -chain }\end{array}$ \\
\hline 1317.1 & 1317.1 & $\mathrm{H}_{4}\left(\mathrm{U}_{7} \mathrm{~V}_{6} \mathrm{P}_{7}\right)(\mathrm{MeCN})_{8}^{3-}$ & $\left\{U_{8} V_{6}\right\}$-dimer \\
\hline 1354.1 & 1354.1 & $\mathrm{H}_{2}(\mathrm{EMIm})_{2}\left[\mathrm{U}_{8}\left(\mathrm{~V}_{3}\right)_{2} \mathrm{P}_{5}\right](\mathrm{MeCN})_{5}\left(\mathrm{H}_{2} \mathrm{O}\right)^{3-}$ & $\left\{U_{8} V_{6}\right\}$-dimer \\
\hline 1421.8 & 1421.8 & $\mathrm{H}(\mathrm{EMIm})_{2}\left(\mathrm{U}_{8} \mathrm{~V}_{6} \mathrm{P}_{8}\right)^{3-}$ & $\begin{array}{l}\left\{U_{8} V_{6}\right\} \text {-monomer } \\
\text {-dimer, -chain }\end{array}$ \\
\hline 1541.2 & 1541.3 & $\mathrm{H}_{2}(\mathrm{EMIm})_{7}\left(\mathrm{U}_{6} \mathrm{~V}_{6} \mathrm{P}_{10}\right)\left(\mathrm{H}_{2} \mathrm{O}\right)_{2}{ }^{3-}$ & $\begin{array}{l}\left\{U_{8} V_{6}\right\} \text {-monomer } \\
\text {-dimer, -chain }\end{array}$ \\
\hline 2086.7 & 2086.7 & $\mathrm{H}_{2}(\mathrm{EMIm})_{3}\left[\mathrm{U}_{8}\left(\mathrm{~V}_{3}\right)_{2} \mathrm{P}_{5}\right](\mathrm{MeCN})_{5}\left(\mathrm{H}_{2} \mathrm{O}\right)^{2-}$ & $\left\{U_{8} V_{6}\right\}$-dimer \\
\hline 2188.3 & 2188.2 & $\mathrm{H}(\mathrm{EMIm})_{3}\left(\mathrm{U}_{8} \mathrm{~V}_{6} \mathrm{P}_{8}\right)^{2-}$ & $\begin{array}{l}\left\{U_{8} V_{6}\right\} \text {-monomer } \\
\text {-dimer, -chain }\end{array}$ \\
\hline
\end{tabular}

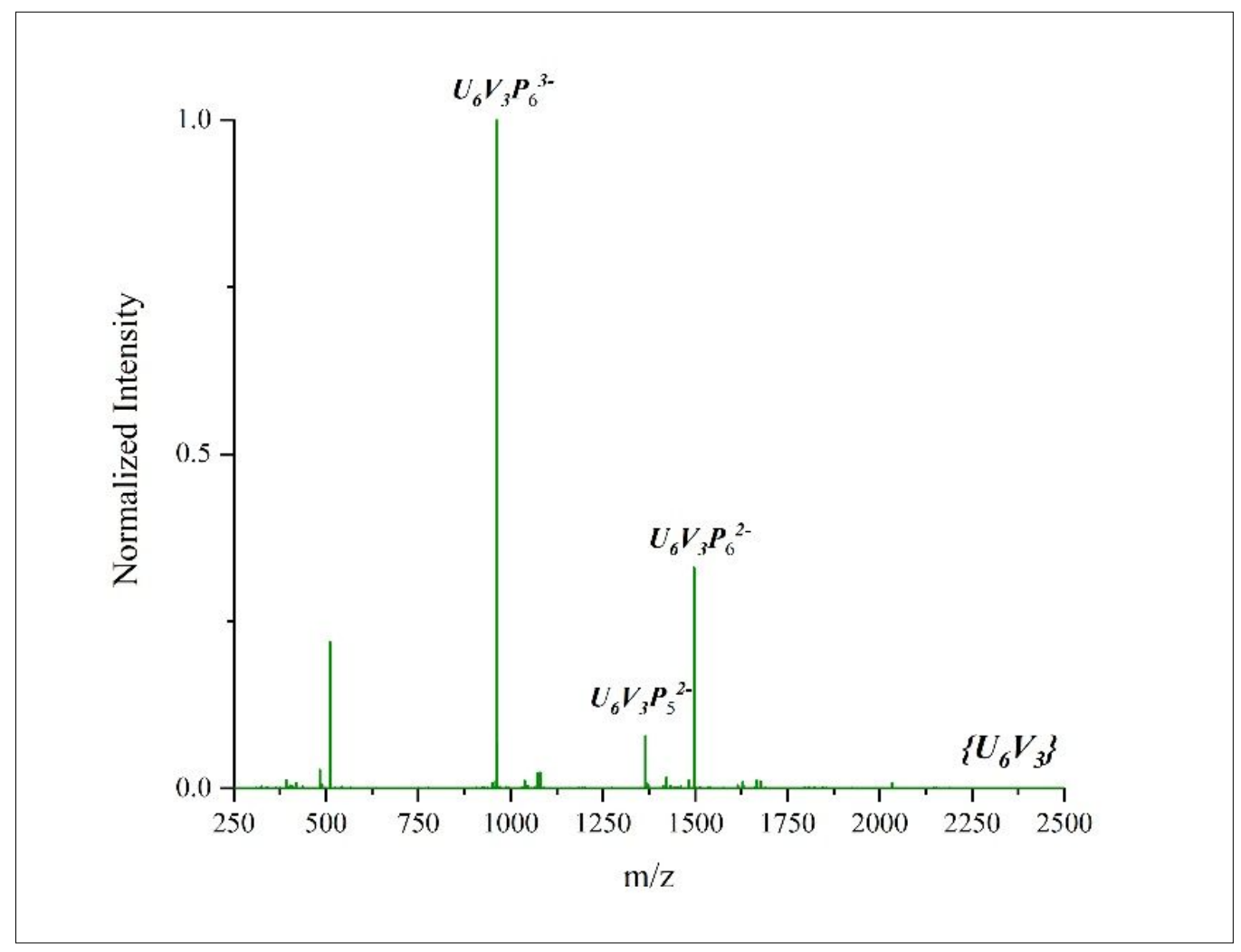




\begin{tabular}{|c|c|c|c|}
\hline Experimental $(\boldsymbol{m} / \mathbf{z})$ & Calculated $(\mathbf{m} / \mathbf{z})$ & Assignment & Materials \\
\hline 511.3 & 512.2 & $\mathrm{H}(\mathrm{EMIm})_{2}(\mathrm{P})(\mathrm{Ph})(\mathrm{MeCN})^{1-}$ & $\left\{U_{6} V_{3}\right\}$ \\
\hline 961.1 & 961.1 & $\mathrm{U}_{6} \mathrm{~V}_{3} \mathrm{P}_{6}{ }^{3-}$ & $\left\{U_{6} V_{3}\right\}$ \\
\hline 1365.1 & 1365.1 & $\mathrm{U}_{6} \mathrm{~V}_{3} \mathrm{P}_{5}{ }^{2-}$ & $\left\{U_{6} V_{3}\right\}$ \\
\hline 1497.2 & 1497.1 & $(\mathrm{EMIm})\left(\mathrm{U}_{6} \mathrm{~V}_{3} \mathrm{P}_{6}\right)^{2-}$ & $\left\{U_{6} V_{3}\right\}$ \\
\hline
\end{tabular}

\section{Day 7 - Acetonitrile}

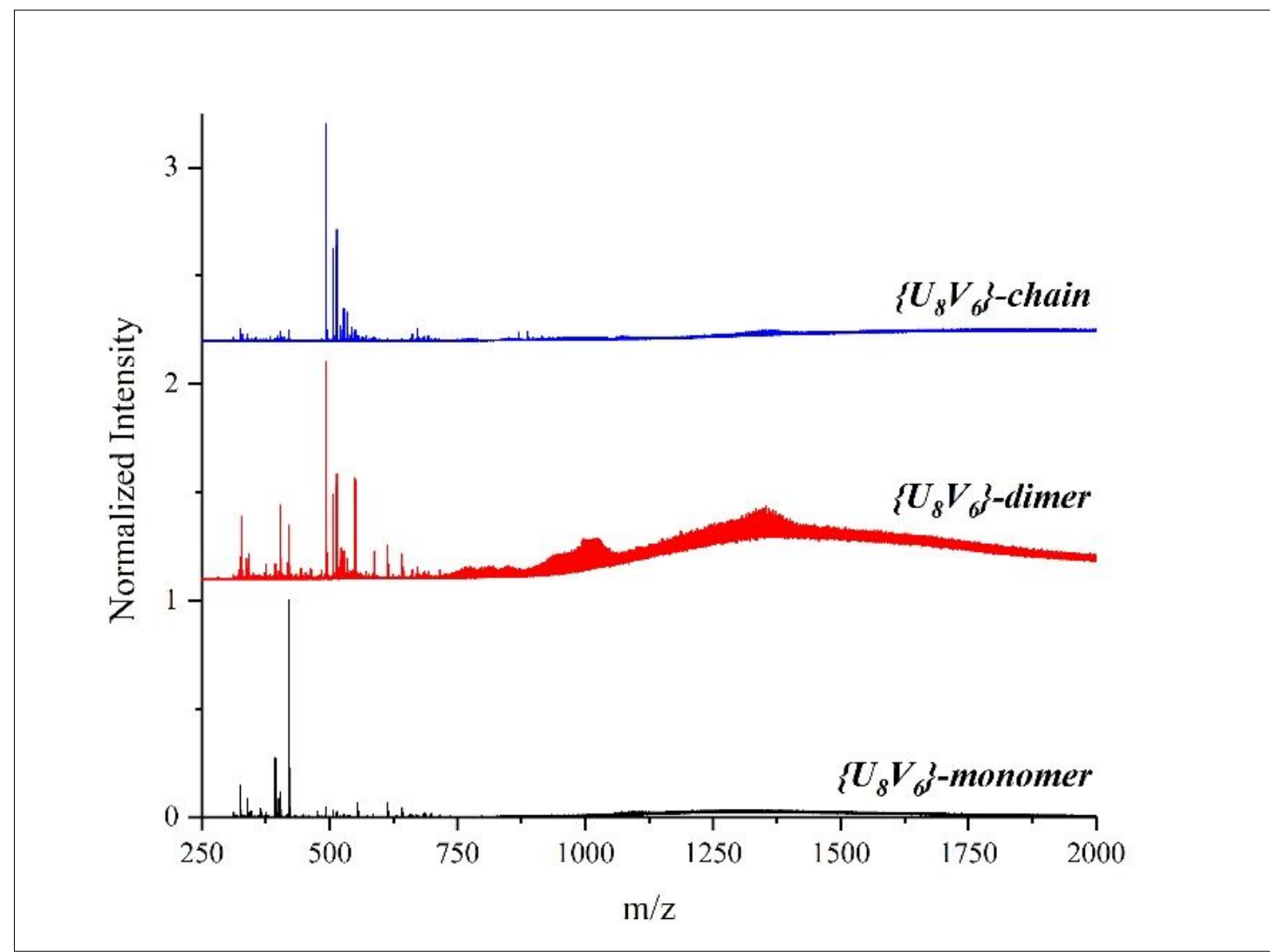

\begin{tabular}{|c|c|c|c|}
\hline Experimental $(\mathbf{m} / \mathbf{z})$ & Calculated & Assignment & Materials \\
\hline & $(\boldsymbol{m} / \mathbf{z})$ & & \\
\hline 325.2 & 325.1 & $\mathrm{H}(\mathrm{EMIm})(\mathrm{Ph})(\mathrm{MeCN})_{2}\left(\mathrm{H}_{2} \mathrm{O}\right)^{1-}$ & $\left\{U_{8} V_{6}\right\}$-monomer, \\
& & & -dimer, -chain \\
\hline
\end{tabular}




\begin{tabular}{|c|c|c|c|}
\hline 393.3 & 394.2 & $(\mathrm{EMIm})_{2}(\mathrm{P})(\mathrm{MeCN})\left(\mathrm{H}_{2} \mathrm{O}\right)_{2}{ }^{1-}$ & $\begin{array}{l}\left\{U_{8} V_{6}\right\} \text {-monomer } \\
\text {-dimer, -chain }\end{array}$ \\
\hline 403.3 & 402.1 & $\mathrm{H}(\mathrm{EMIm})(\mathrm{P})(\mathrm{Ph})(\mathrm{MeCN})^{1-}$ & $\begin{array}{l}\left\{U_{8} V_{6}\right\} \text {-monomer } \\
\text {-dimer, -chain }\end{array}$ \\
\hline 420.3 & 420.1 & $\mathrm{H}(\mathrm{EMIm})(\mathrm{P})(\mathrm{Ph})(\mathrm{MeCN})\left(\mathrm{H}_{2} \mathrm{O}\right)^{1-}$ & $\begin{array}{l}\left\{U_{8} V_{6}\right\} \text {-monomer } \\
\text {-dimer, -chain }\end{array}$ \\
\hline 492.8 & 493.09 & $\mathrm{H}(\mathrm{EMIm})_{2}\left(\mathrm{U}_{2} \mathrm{Ph}_{2}\right)\left(\mathrm{H}_{2} \mathrm{O}\right)^{2-}$ & $\begin{array}{l}\left\{U_{8} V_{6}\right\} \text {-monomer } \\
\text {-dimer, -chain }\end{array}$ \\
\hline 513.8 & 513.1 & $(\mathrm{EMIm})_{2}\left(\mathrm{UP}_{2} \mathrm{Ph}_{2}\right)\left(\mathrm{H}_{2} \mathrm{O}\right)_{2}{ }^{2-}$ & $\begin{array}{l}\left\{U_{8} V_{6}\right\} \text {-monomer } \\
\text {-dimer, -chain }\end{array}$ \\
\hline 549.9 & 550.4 & $(\mathrm{EMIm})_{2}\left(\mathrm{U}_{2} \mathrm{~V}_{3} \mathrm{P}_{3}\right)(\mathrm{MeCN})_{2}^{3-}$ & $\begin{array}{l}\left\{U_{8} V_{6}\right\} \text {-monomer } \\
\text {-dimer, -chain }\end{array}$ \\
\hline
\end{tabular}




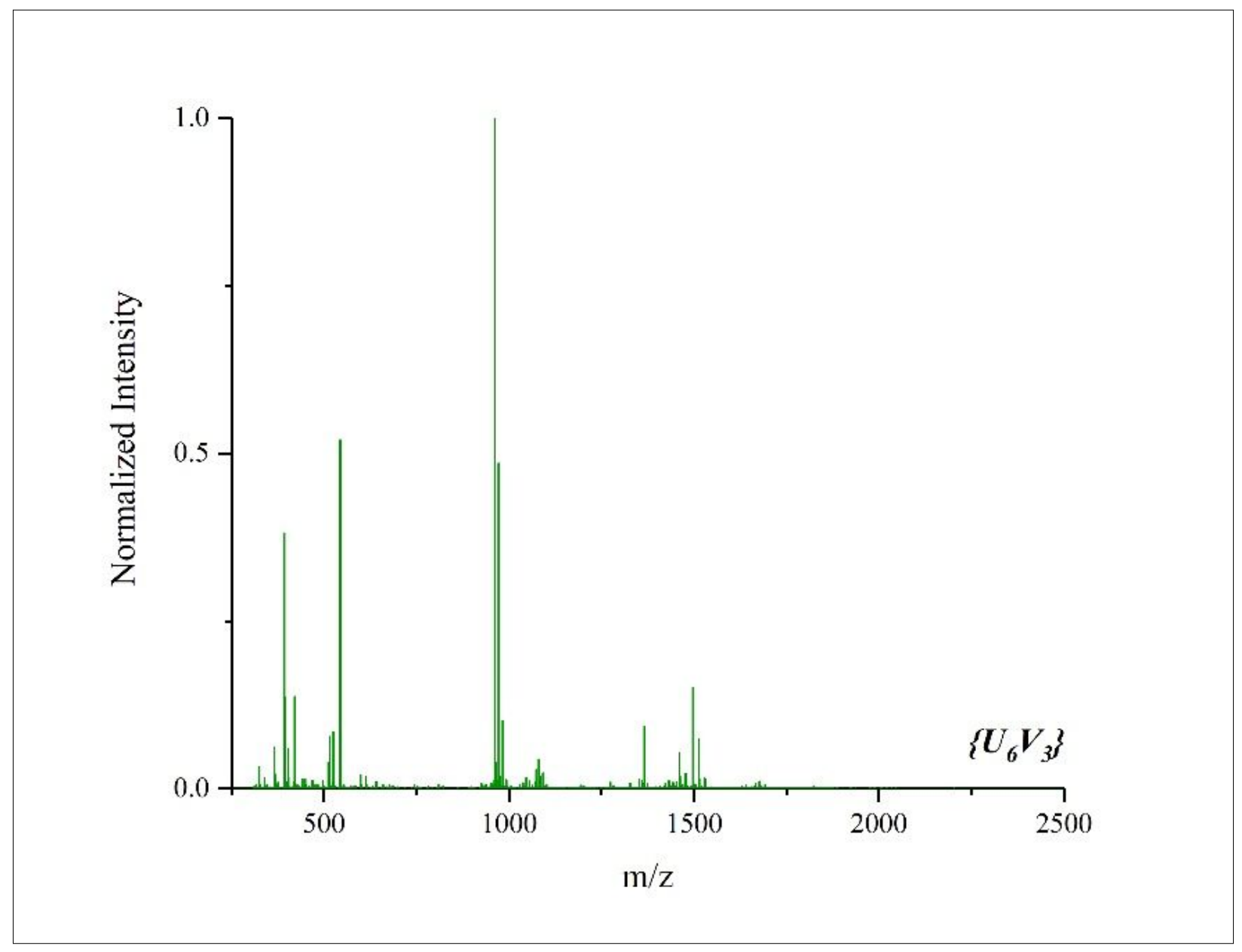

\begin{tabular}{|c|c|c|c|}
\hline Experimental $(\mathbf{m} / \mathbf{z})$ & Calculated & Assignment & Materials \\
\hline 393.3 & $(\boldsymbol{m} / z)$ & $(\mathrm{EMIm})_{2}(\mathrm{P})(\mathrm{MeCN})\left(\mathrm{H}_{2} \mathrm{O}\right)_{2}{ }^{1-}$ & $\left\{U_{6} V_{3}\right\}$ \\
\hline 543.2 & 394.2 & $\mathrm{H}_{2}\left(\mathrm{P}_{3}\right)(\mathrm{MeCN})_{2}{ }^{1-}$ & $\left\{U_{6} V_{3}\right\}$ \\
\hline 961.1 & 543.2 & $\mathrm{U}_{6} \mathrm{~V}_{3} \mathrm{P}_{6}{ }^{3-}$ & $\left\{U_{6} V_{3}\right\}$ \\
\hline 971.7 & 961.1 & $\mathrm{H}(\mathrm{EMIm})_{3}\left[\mathrm{U}_{6}\left(\mathrm{Va}_{3}\right)_{2} \mathrm{P}\right](\mathrm{MeCN})_{2}{ }^{3-}$ & $\left\{U_{6} V_{3}\right\}$ \\
\hline 1365.1 & 971.7 & $\mathrm{U}_{6} \mathrm{~V}_{3} \mathrm{P}_{5}{ }^{2-}$ & $\left\{U_{6} V_{3}\right\}$ \\
\hline 1497.2 & 1365.1 & $\left(\mathrm{EMIm}_{3}\right)\left(\mathrm{U}_{6} \mathrm{~V}_{3} \mathrm{P}_{6}\right)^{2-}$ & $\left\{U_{6} V_{3}\right\}$ \\
\hline
\end{tabular}


Figure S6. Experimental powder X-ray diffraction (pXRD) pattern from harvested material compared to the simulated pXRD pattern from scXRD data using the CrystalDiffract software. The pXRD patterns were collected using a Bruker D8 DAVINCI Advance diffractometer equipped with a $\mathrm{Cu} K_{\alpha} \mathrm{X}$-ray source $(1.5406 \AA)$ with parameters listed below.

$\left\{U_{8} V_{6}\right\}$-monomer $+\left\{U_{8} V_{6}\right\}$-chain synthesis.

Parameters for collection

$3-20^{\circ} 2 \theta / 0.019$ step size / 3.25 sec per step / $10 \mathrm{rpm}$

pXRD pattern of the experimental material shows dominant phase as $\left\{\boldsymbol{U}_{\boldsymbol{\delta}} \boldsymbol{V}_{\boldsymbol{b}}\right\}$-chain with minor contribution from $\left\{U_{8} V_{6}\right\}$-monomer.

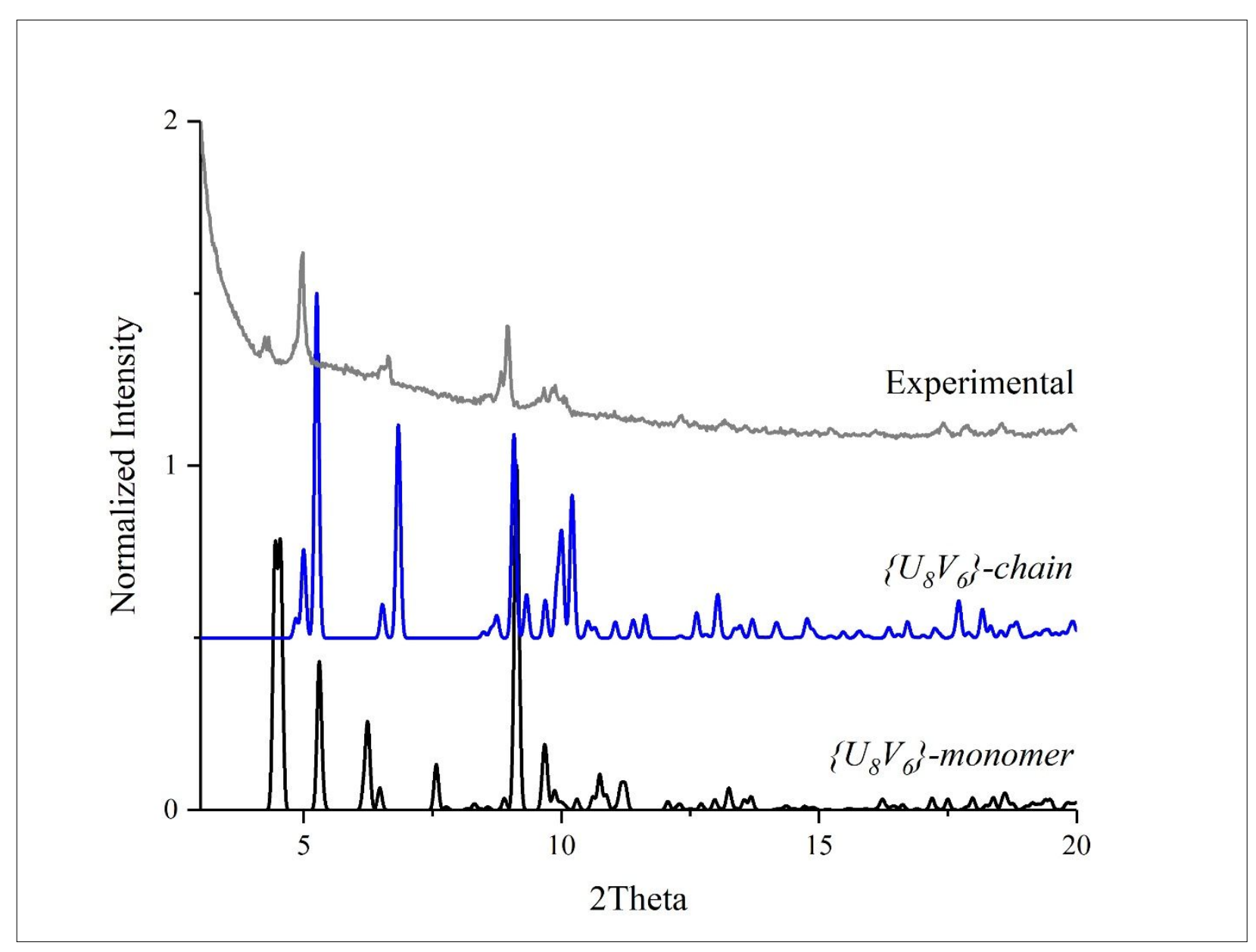


$\left\{U_{8} V_{b}\right\}$-dimer + side product synthesis.

Parameters for collection

$3-20^{\circ} 2 \theta / 0.019$ step size / $3.25 \mathrm{sec}$ per step / $10 \mathrm{rpm}$

pXRD pattern of the experimental material shows dominant phase as $\left\{\boldsymbol{U}_{\boldsymbol{\delta}} \boldsymbol{V}_{\boldsymbol{\sigma}}\right\}$-dimer with little to no contribution from side product, although optically it is known to be present.

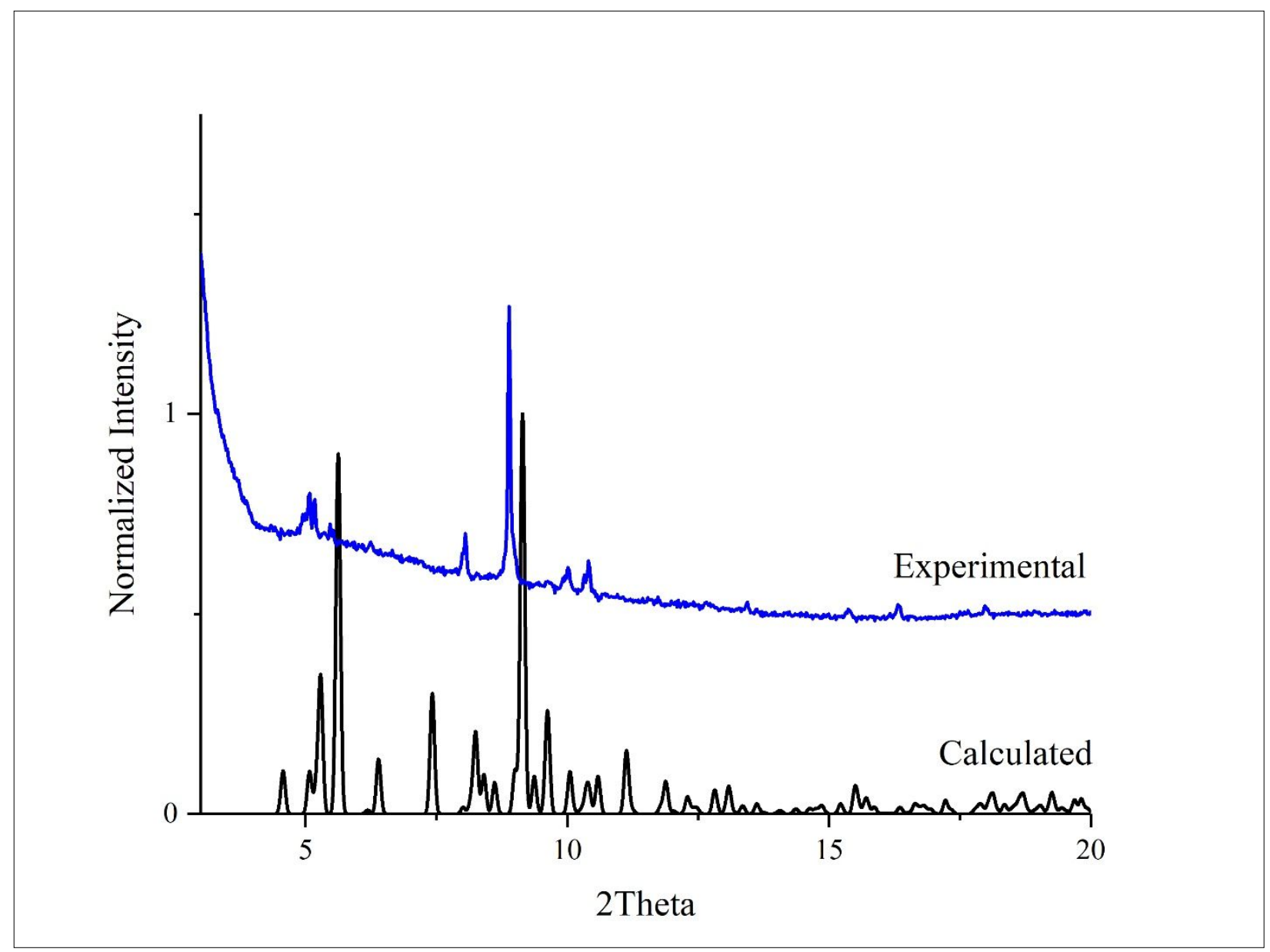


$\left\{\boldsymbol{U}_{\boldsymbol{\delta}} \boldsymbol{V}_{\boldsymbol{G}}\right\}$-chain + side product synthesis.

Parameters for collection

$4-40^{\circ} 2 \theta / 0.02$ step size / $1.75 \mathrm{sec}$ per step / $10 \mathrm{rpm}$

pXRD pattern of the experimental material shows dominant phase as $\left\{\boldsymbol{U}_{\boldsymbol{\delta}} \boldsymbol{V}_{\boldsymbol{6}}\right\}$-chain with little to no contribution from side product, although optically it is known to be present.

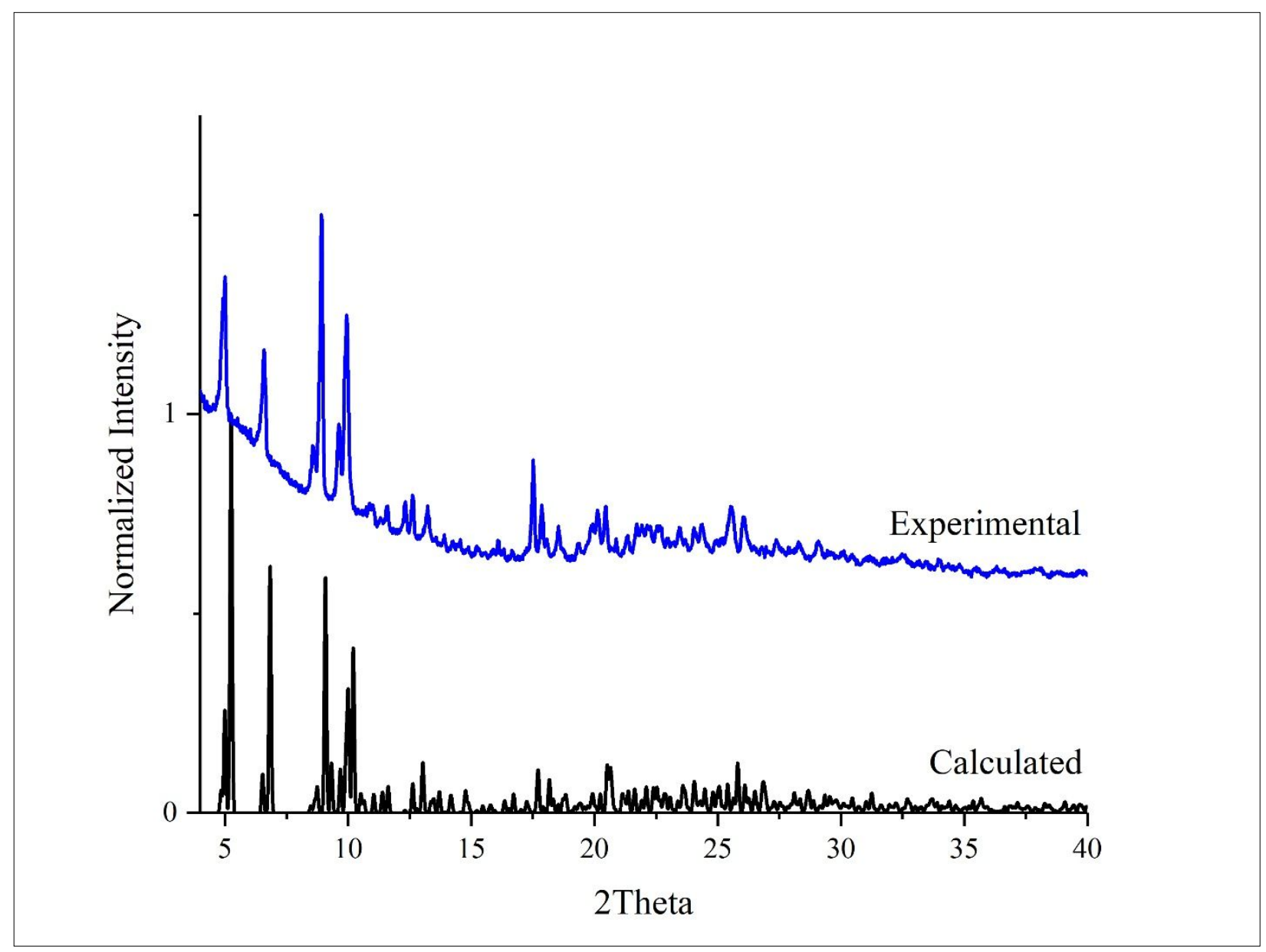


$\left\{U_{6} V_{3}\right\}+$ precipitate.

Parameters for collection

$3-20^{\circ} 2 \theta / 0.019$ step size / $3.25 \mathrm{sec}$ per step / $10 \mathrm{rpm}$

pXRD pattern of the experimental material shows dominant phase as $\left\{\boldsymbol{U}_{6} \boldsymbol{V}_{3}\right\}$ but with poor to no diffraction at higher angle.

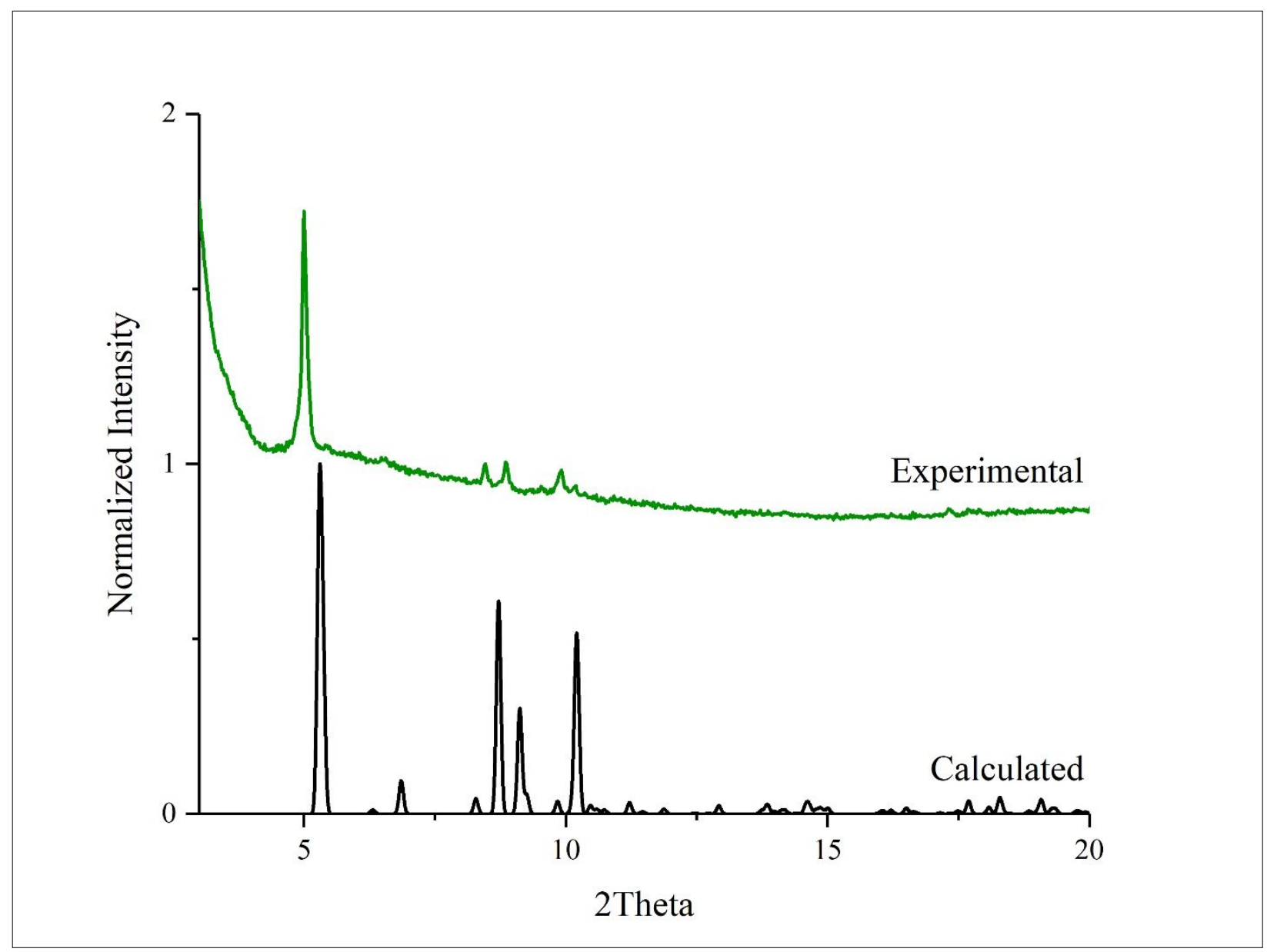


Figure S7. Thermogravimetric analysis (TGA) and CHN elemental analysis data of harvested $\left\{\boldsymbol{U}_{\boldsymbol{8}} \boldsymbol{V}_{\boldsymbol{6}}\right\}$-chain material, which was shown to be phase pure by experimental powder X-ray diffraction (pXRD) in Figure S6. It was noted that there was visible contamination of byproduct under a light microscope of the harvested material, which the impurity phases were not detected by the experimental $\mathrm{pXRD}$ pattern. The $\mathrm{pXRD}$ pattern of the material post-TGA analysis was also studied. TGA was done using a TA Instruments Q50. Approximately $11 \mathrm{mg}$ of sample was measured into an alumina crucible and was heated to $900{ }^{\circ} \mathrm{C}$ at a ramp rate of $10{ }^{\circ} \mathrm{C} / \mathrm{min}$ with a $\mathrm{N}_{2}$ gas flow. CHN elemental analysis was performed using a Costech ECS 4010 EA gas chromatograph.

$\mathrm{CHN}$ analysis showed $\mathrm{N}$ as 3.39 weight $\%$, while $\mathrm{C}$ was 16.62 weight $\%$. The theoretical values for each are 3.44 weight $\% \mathrm{~N}$ and 17.7 weight $\% \mathrm{C}$. The slight differences are attributed to the impurity phase. The TGA curve shows a weight loss of $26.4 \%$. The theoretical weight loss of sodium, EMIm cations, and ethyl groups from the diethyl phosphate would be 24.83 weight $\%$. The remaining theoretical 75.17 weight $\%$ is attributed to uranium oxide, vanadate, and phosphate phases. The pXRD of post-TGA material had a match for $\mathrm{UO}_{2}$ in the crystallographic database, but peaks below $25^{\circ} 2 \theta$ were not matched to any pattern in the crystallographic database. They are assumed to attributed to unidentified phosphate- and vanadate-based phases. 
CHN Elemental Analysis Results

\begin{tabular}{|l|c|c|c|}
\hline & Weight \% N & Weight \% C & Carbon Response \\
& & & Ratio \\
\hline $\mathbf{1}$ & 3.16 & 15.55 & 0.074 \\
\hline $\mathbf{2}$ & 3.50 & 16.90 & 0.076 \\
\hline $\mathbf{3}$ & 3.52 & 17.41 & 0.073 \\
\hline Average & $\mathbf{3 . 3 9}$ & $\mathbf{1 6 . 6 2}$ & 0.074 \\
\hline Theoretical & $\mathbf{3 . 4 4}$ & $\mathbf{1 7 . 7}$ & - \\
\hline
\end{tabular}

TGA Curve

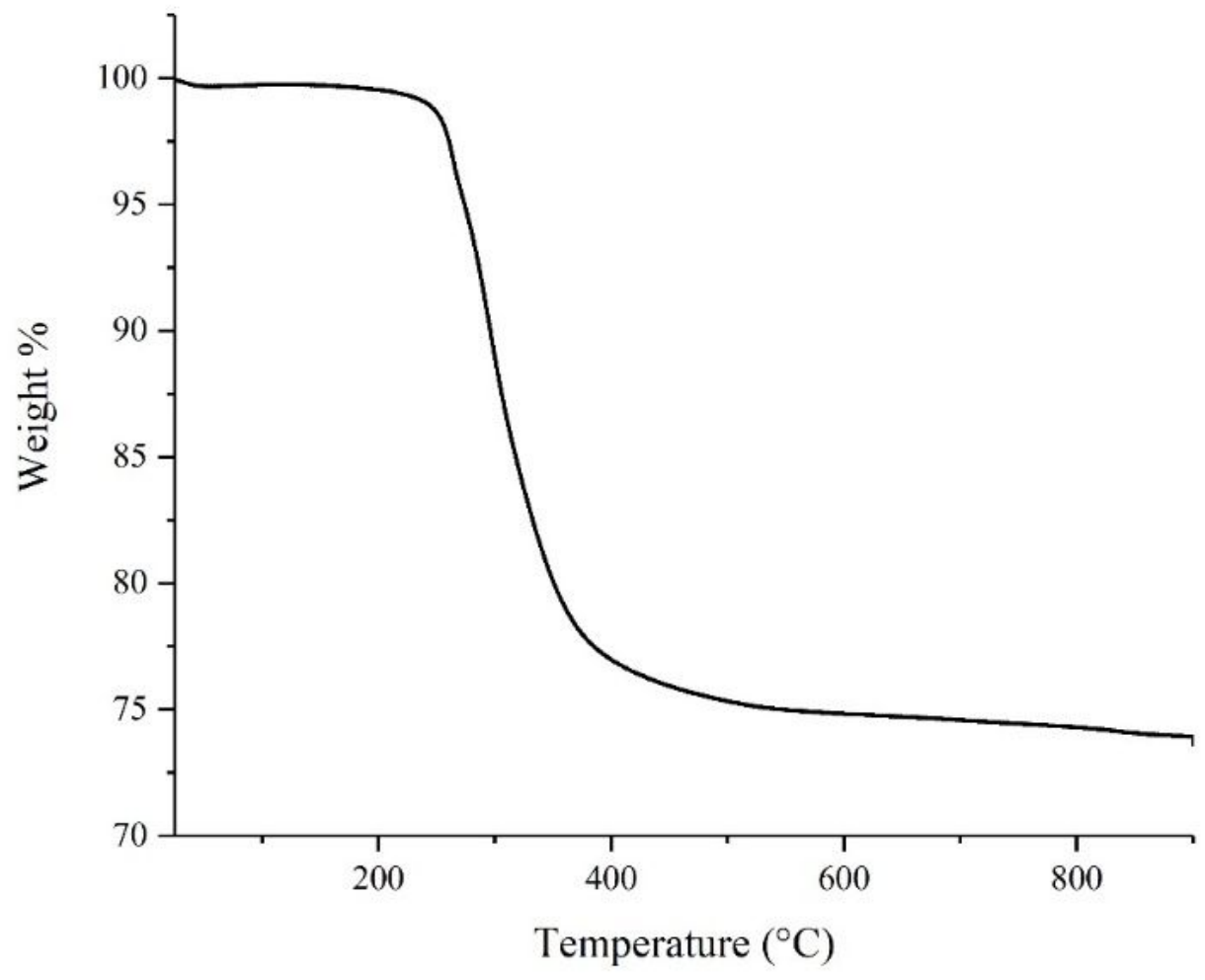




\section{pXRD of material post-TGA analysis}

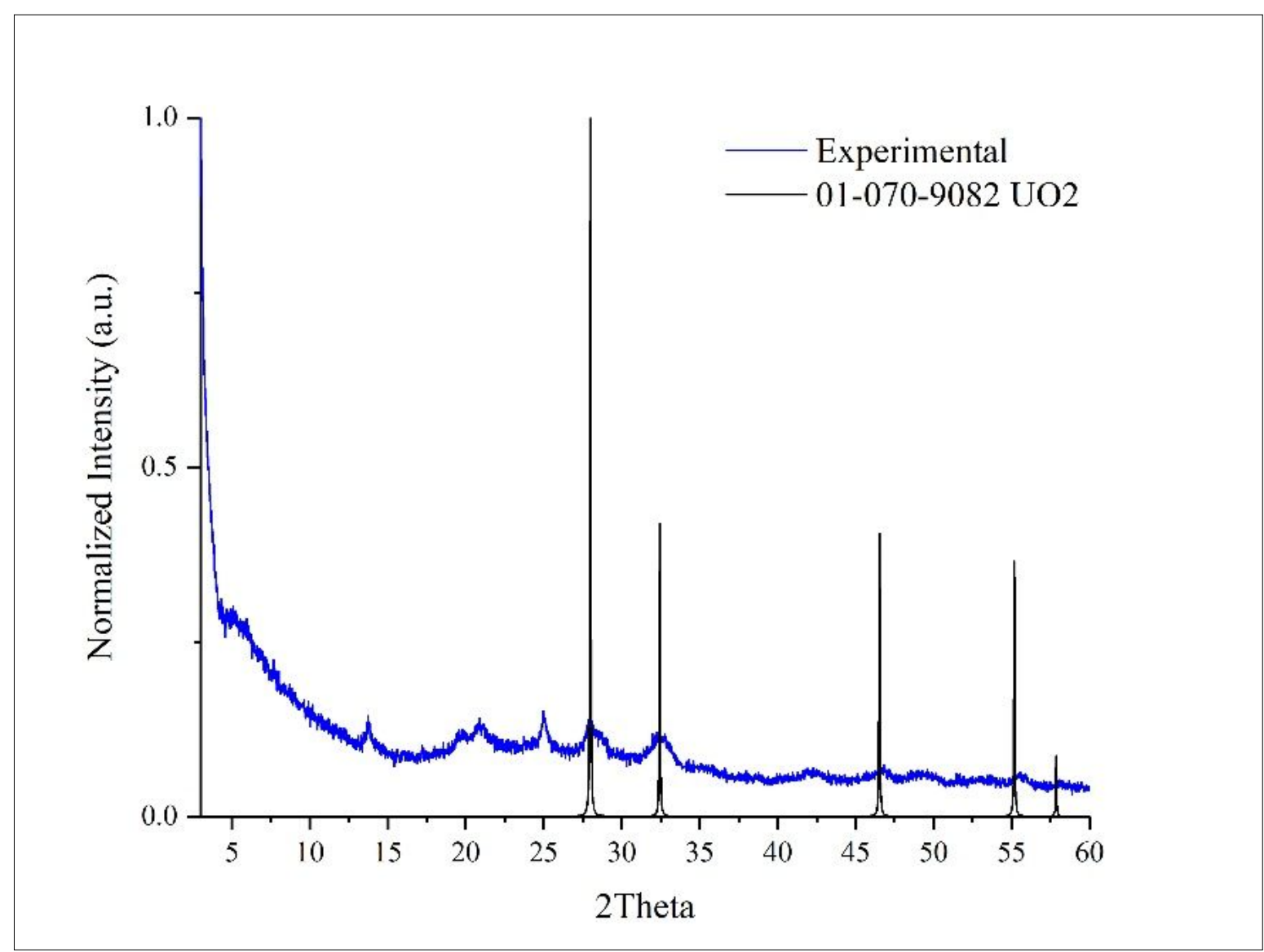


Figure S8. Keggin-type POM structure. Due to poor scXRD data, only this structural unit along with one EMIm counter cation was resolved. Based on the low quality scXRD data and charge balance requirements of the POM, the proposed chemical formula is $\left(\mathrm{C}_{6} \mathrm{~N}_{2} \mathrm{H}_{11}\right)_{3}\left[\mathrm{PV}_{16} \mathrm{O}_{44}\right]$. Green polyhedra are vanadium in octahedral coordination, faded green polyhedra are vanadium in square pyramidal coordination, and magenta is phosphorus.

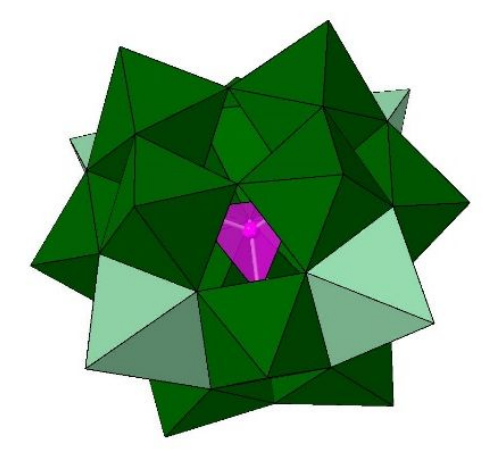

Figure S9. (a) Decavanadate $\left\{\boldsymbol{V}_{10}\right\}$ POM and (b) the extended structure of decavanadate viewed down the $y$-axis. The proposed chemical formula for this product is $\left(\mathrm{C}_{6} \mathrm{~N}_{2} \mathrm{H}_{11}\right)_{7.5} \mathrm{Na}_{0.5}\left\{\left[\mathrm{~V}_{10} \mathrm{O}_{26}(\mathrm{OH})_{2}\right]_{2}\right\}$ based on the scXRD data and bond valence summations presented in Table S6. Green polyhedra are vanadium, blue spheres are nitrogen, black spheres are carbon, and yellow spheres are sodium. The hydrogen atoms have been omitted for clarity.

(a)

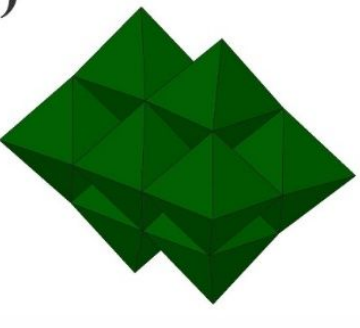

(b)

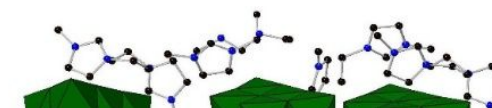

,
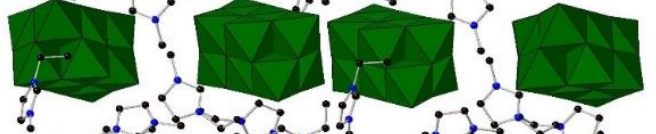

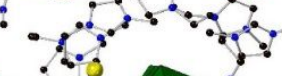

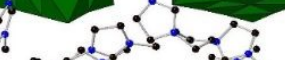

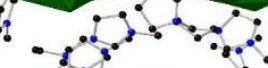

0

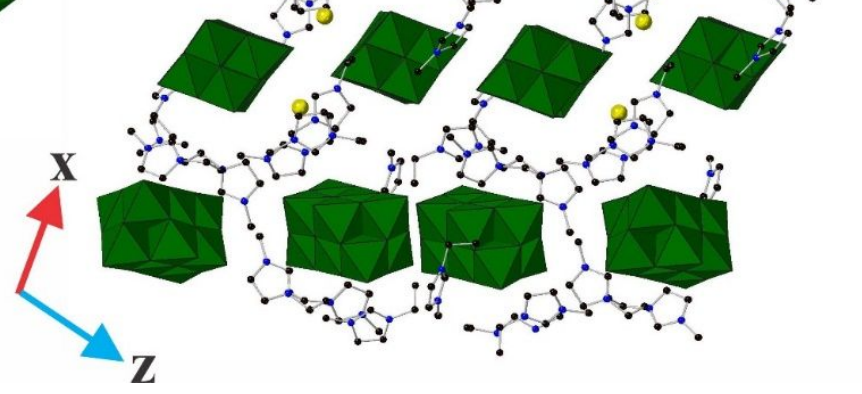


Table S1. Summary of the observed reaction products. When possible, products were verified by X-ray diffraction.

\begin{tabular}{|c|c|c|c|c|}
\hline & \multicolumn{2}{|c|}{$\begin{array}{c}\text { System } \\
\mathrm{UO}_{2}\left(\mathrm{NO}_{3}\right)_{2} / \mathrm{NaVO} \mathrm{NOSO}_{4} / \mathrm{VOSO}_{4}\end{array}$} & \multicolumn{2}{|c|}{$\begin{array}{c}\text { System } \\
\mathrm{UO}_{2}\left(\mathrm{NO}_{3}\right)_{2} / \mathrm{Na}_{3} \mathrm{VO}_{4} / \mathrm{VOSO}_{4} \\
\end{array}$} \\
\hline Volumes & Ratio & Result & Ratio & Result \\
\hline \multirow{6}{*}{$\begin{array}{c}\text { Normal } \\
\text { Volume } \\
(0.25 / 0.5 \\
\text { mL IL) }\end{array}$} & $1: 1: 0$ & $\begin{array}{c}\left\{U_{8} V_{6\}}\right\} \text {-monomer } \\
\left\{U_{8} V_{6}\right\} \text {-chain }\end{array}$ & $1: 1: 0$ & $\begin{array}{c}\left\{\boldsymbol{U}_{8} \boldsymbol{V}_{6}\right\} \text {-chain } \\
\text { Microcrystal/Precipitate }\end{array}$ \\
\hline & $1: 2: 0$ & $\begin{array}{c}\left\{\boldsymbol{U}_{\boldsymbol{B}} \boldsymbol{V}_{\boldsymbol{6}}\right\} \text {-chain } \\
\text { Black and/or orange material }\end{array}$ & $1: 2: 0$ & Microcrystal/Precipitate \\
\hline & $2: 1: 0$ & $\begin{array}{c}\left\{U_{\delta} V_{6}\right\} \text {-monomer } \\
\text { Microcrystals/Precipitate }\end{array}$ & $2: 1: 0$ & $\begin{array}{c}\left\{\boldsymbol{U}_{6} \boldsymbol{V}_{3}\right\} \\
\text { Microcrystal/Precipitate } \\
\end{array}$ \\
\hline & $1: 1: 1$ & $\begin{array}{l}\left\{U_{8} V_{6\}}\right\} \text {-dimer } \\
\text { Green material }\end{array}$ & $1: 1: 1$ & $\begin{array}{l}\left\{U_{8} V_{6}\right\} \text {-dimer } \\
\text { Green material }\end{array}$ \\
\hline & $2: 1: 1$ & $\begin{array}{c}\left\{U_{8} V_{6\}}\right\} \text {-monomer } \\
\text { Green material }\end{array}$ & $2: 1: 1$ & $\left\{U_{6} V_{3}\right\} /\left\{U_{8} V_{6}\right\}$-monomer $?$ \\
\hline & 4:1:1 & $\left\{U_{8} V_{6}\right\}$-monomer? & $4: 1: 1$ & $\left\{U_{6} V_{3}\right\} /\left\{U_{8} V_{6}\right\}$-monomer? \\
\hline Volumes & Ratio & Result & Ratio & Result \\
\hline \multirow{6}{*}{$\begin{array}{c}2 \mathrm{x} \\
\text { Volume of } \\
\text { all } \\
\text { solutions }\end{array}$} & $1: 1: 0$ & $\begin{array}{c}\left\{U_{8} V_{6}\right\} \text {-monomer } \\
\left\{U_{8} V_{6}\right\} \text {-chain }\end{array}$ & $1: 1: 0$ & $\begin{array}{c}\left\{\boldsymbol{U}_{\boldsymbol{\delta}} \boldsymbol{V}_{6}\right\} \text {-chain } \\
\text { Microcrystal/Precipitate }\end{array}$ \\
\hline & $1: 2: 0$ & $\begin{array}{c}\left\{\boldsymbol{U}_{\boldsymbol{B}} \boldsymbol{V}_{\boldsymbol{\sigma}}\right\} \text {-chain } \\
\text { Black and/or orange material }\end{array}$ & $1: 2: 0$ & Microcrystal/Precipitate \\
\hline & $2: 1: 0$ & No crystals & $2: 1: 0$ & No crystals \\
\hline & $1: 1: 1$ & $\begin{array}{c}\left\{U_{8} V_{6 j}\right\} \text {-dimer } \\
\text { Green material }\end{array}$ & $1: 1: 1$ & $\begin{array}{c}\left\{U_{6} V_{3}\right\} \\
\left\{U_{8} V_{6}\right\} \text {-dimer } \\
\text { Green material }\end{array}$ \\
\hline & $2: 1: 1$ & $\begin{array}{l}\left\{U_{8} V_{6}\right\} \text {-dimer } \\
\text { Green material }\end{array}$ & $2: 1: 1$ & $\begin{array}{c}\left\{\boldsymbol{U}_{6} \boldsymbol{V}_{3}\right\} \\
\text { Green material }\end{array}$ \\
\hline & 4:1:1 & No crystals & $4: 1: 1$ & $\left\{U_{6} V_{3}\right\} /\left\{U_{8} V_{6}\right\}$-monomer $?$ \\
\hline
\end{tabular}


Table S2. Summary of the crystallographic data and refinement parameters.

\begin{tabular}{|c|c|c|c|c|}
\hline Crystal Data & $\left\{U_{8} V_{6}\right\}$-monomer & $\left\{U_{8} V_{6}\right\}$-dimer & $\left\{U_{8} V_{6}\right\}$-chain & $\left\{U_{6} V_{3}\right\}-W$ \\
\hline Formula weight & 4756.38 & 4397.19 & 4885.68 & 3783.29 \\
\hline Temperature $(\mathrm{K})$ & $200(2)$ & $200(2)$ & $200(2)$ & $200(2)$ \\
\hline Crystal system & Monoclinic & Triclinic & Orthorhombic & Monoclinic \\
\hline Space group & $P 2_{1} / n$ & $P-1$ & $P_{n a 2}$ & $P 2{ }_{1} / c$ \\
\hline$a(\AA)$ & $27.277(3)$ & 18.999(3) & $20.196(3)$ & $19.393(2)$ \\
\hline$b(\AA)$ & $20.609(2)$ & $20.085(3)$ & $36.426(5)$ & $29.230(3)$ \\
\hline$c(\AA)$ & $28.302(3)$ & $21.300(3)$ & $18.948(3)$ & $19.657(2)$ \\
\hline$\alpha\left(^{\circ}\right)$ & 90 & $112.736(2)$ & 90 & 90 \\
\hline$\beta\left(^{\circ}\right)$ & $91.3225(16)$ & $90.808(2)$ & 90 & $97.5696(15)$ \\
\hline$\gamma\left({ }^{\circ}\right)$ & 90 & $111.4257(19)$ & 90 & 90 \\
\hline$V\left(\AA^{3}\right)$ & $15906(3)$ & $6863.3(18)$ & $13940(3)$ & $11045(2)$ \\
\hline$Z$ & 4 & 2 & 4 & 4 \\
\hline$\rho_{\text {calc }}\left(\mathrm{g} \mathrm{cm}^{-3}\right)$ & 1.986 & 2.128 & 2.328 & 2.275 \\
\hline$\mu\left(\mathrm{mm}^{-1}\right)$ & 8.633 & 9.979 & 9.842 & 10.169 \\
\hline
\end{tabular}




\begin{tabular}{|c|c|c|c|c|}
\hline $\mathrm{F}(000)$ & 8568 & 3926 & 9136 & 7056 \\
\hline Data Range $\theta\left(^{\circ}\right)$ & $2.23-20.41$ & $2.21-24.22$ & $2.29-27.75$ & $2.23-27.90$ \\
\hline $\begin{array}{l}\text { Total Reflections } \\
\text { Collected }\end{array}$ & 130576 & 84922 & 168084 & 136353 \\
\hline $\begin{array}{l}\text { Independent } \\
\text { Reflections / } \\
\text { Parameters / } \\
\text { Restraints }\end{array}$ & $\begin{array}{c}21798 / \\
971 / \\
177\end{array}$ & $\begin{array}{c}32891 / \\
899 / \\
247\end{array}$ & $\begin{array}{c}34850 / \\
1,638 / \\
2\end{array}$ & $\begin{array}{c}27961 / \\
916 / \\
132\end{array}$ \\
\hline$R_{\text {int }}(\%)$ & 10.53 & 7.33 & 5.32 & 5.72 \\
\hline$S^{a}$ & 1.022 & 1.053 & 1.030 & 1.024 \\
\hline$R_{1}, w R_{2}[I>2 \sigma(I)]^{b, c}$ & $7.46,19.67$ & $8.97,24.56$ & $3.50,7.19$ & $4.04,9.34$ \\
\hline$R_{1}, w R_{2}(\text { all data })^{b, c}$ & $13.48,24.13$ & $17.42,29.52$ & $4.68,7.51$ & $6.88,10.48$ \\
\hline
\end{tabular}

${ }^{a} S=\mathrm{GooF}=\left[\Sigma w\left(F_{o}^{2}-F_{c}^{2}\right)^{2} /(n-p)\right]^{1 / 2}$

${ }^{b} R_{1}=\Sigma|| F_{o}|-| F_{c}|| / \Sigma\left|F_{o}\right|$

${ }^{c} w R_{2}=\left[\Sigma w\left(F_{o}^{2}-F_{c}^{2}\right)^{2} / \Sigma w\left(F_{o}^{2}\right)^{2}\right]^{1 / 2}$

$w=1 /\left(\sigma^{2}\left(F_{o}^{2}\right)+(a P)^{2}+b P\right)$ where $P=\left(\max \left(F_{o}^{2}, 0\right)+2 F_{c}^{2}\right) / 3$ 
Table S3. Bond valence summations (BVS) for cations uranium, vanadium, and phosphorus, and BVS for oxygen anions of the structural unit in $\left\{\boldsymbol{U}_{\boldsymbol{\delta}} \boldsymbol{V}_{\boldsymbol{6}}\right\}$-monomer. BVS for oxygen atoms that are highlighted in orange-yellow are inferred to have a missing C(IV)$\mathrm{O}$ bond, so their BVS is expectedly low. The $\mathrm{C}$ atoms were not located in the difference Fourier map. BVS for oxygen atoms that are highlighted in green are inferred to be hydroxide oxygen atoms. The $\mathrm{H}$ atoms were not located in the difference Fourier map.

\begin{tabular}{|c|c|c|c|c|c|c|c|c|}
\hline Site & $\mathbf{U 1}$ & $\mathbf{U 2}$ & $\mathbf{U 3}$ & $\mathbf{U 4}$ & $\mathbf{U 5}$ & $\mathbf{U 6}$ & $\mathbf{U 7}$ & $\mathbf{U 8}$ \\
\hline $\mathbf{O}$ & 1.79 & 1.71 & 1.70 & 2.08 & 1.83 & 1.67 & 1.71 & 1.71 \\
\hline $\mathbf{O}$ & 1.68 & 1.62 & 1.65 & 1.68 & 1.80 & 1.86 & 1.70 & 1.74 \\
\hline $\mathbf{O}$ & 0.55 & 0.57 & 0.56 & 0.57 & 0.56 & 0.59 & 0.57 & 0.58 \\
\hline $\mathbf{O}$ & 0.59 & 0.56 & 0.60 & 0.57 & 0.56 & 0.55 & 0.57 & 0.57 \\
\hline $\mathbf{O}$ & 0.60 & 0.55 & 0.55 & 0.55 & 0.50 & 0.57 & 0.57 & 0.55 \\
\hline $\mathbf{O}$ & 0.50 & 0.54 & 0.53 & 0.55 & 0.61 & 0.57 & 0.53 & 0.53 \\
\hline $\mathbf{O}$ & 0.53 & 0.52 & 0.52 & 0.58 & 0.53 & 0.53 & 0.53 & 0.59 \\
\hline $\boldsymbol{V}_{\boldsymbol{c}}$ & 6.25 & 6.07 & 6.11 & 6.59 & 6.39 & 6.34 & 6.17 & 6.26 \\
\hline
\end{tabular}

\begin{tabular}{|c|c|c|c|c|c|c|}
\hline Site & $\mathbf{V 1}$ & $\mathbf{V 2}$ & $\mathbf{V 3}$ & $\mathbf{V 4}$ & $\mathbf{V 5}$ & $\mathbf{V 6}$ \\
\hline $\mathbf{O}$ & 1.98 & 1.69 & 1.76 & 1.80 & 1.68 & 1.80 \\
\hline $\mathbf{O}$ & 0.74 & 0.75 & 0.71 & 0.72 & 0.73 & 0.73 \\
\hline $\mathbf{O}$ & 0.76 & 0.90 & 0.82 & 0.78 & 0.89 & 0.76 \\
\hline $\mathbf{O}$ & 0.96 & 0.75 & 0.94 & 0.94 & 0.84 & 0.90 \\
\hline $\mathbf{O}$ & 0.96 & 0.92 & 1.07 & 1.02 & 0.94 & 0.97 \\
\hline $\boldsymbol{V}_{\boldsymbol{c}}$ & 5.41 & 5.02 & 5.29 & 5.26 & 5.08 & 5.16 \\
\hline
\end{tabular}




\begin{tabular}{|c|c|c|c|c|c|c|c|c|c|c|}
\hline Site & P1 & P2 & P3 & P4 & P5 & P6 & P7 & P8 & P9 & P10 \\
\hline $\mathbf{O}$ & 1.30 & 1.61 & 1.62 & 1.51 & 1.34 & 1.23 & 1.45 & 1.52 & 1.42 & 1.37 \\
\hline $\mathbf{O}$ & 1.20 & 1.53 & 1.44 & 1.53 & 1.57 & 1.05 & 1.42 & 1.43 & 1.53 & 1.50 \\
\hline $\mathbf{O}$ & 1.66 & 1.17 & 1.11 & 1.34 & 1.30 & 0.61 & 1.02 & 1.14 & 1.20 & 1.14 \\
\hline $\mathbf{O}$ & 0.84 & 1.08 & 1.11 & 0.94 & 1.57 & 0.49 & 1.02 & 1.41 & 1.08 & 0.99 \\
\hline $\boldsymbol{V}_{\boldsymbol{c}}$ & 5.00 & 5.39 & 5.27 & 5.32 & 5.78 & 3.38 & 4.91 & 5.50 & 5.23 & 5.00 \\
\hline
\end{tabular}

\begin{tabular}{|c|c|c|c|c|c|c|c|c|c|c|c|}
\hline Site & $\mathbf{O 1}$ & $\mathbf{O 2}$ & $\mathbf{O 3}$ & $\mathbf{O 4}$ & $\mathbf{O 5}$ & $\mathbf{O 6}$ & $\mathbf{O 7}$ & $\mathbf{O 8}$ & $\mathbf{O 9}$ & $\mathbf{O 1 0}$ & $\mathbf{O 1 1}$ \\
\hline $\boldsymbol{V}_{\boldsymbol{a}}$ & 1.79 & 1.68 & 1.71 & 1.62 & 1.70 & 1.65 & 2.08 & 1.68 & 1.83 & 1.80 & 1.67 \\
\hline Site & $\mathbf{O 1 2}$ & $\mathbf{O 1 3}$ & $\mathbf{O 1 4}$ & $\mathbf{O 1 5}$ & $\mathbf{O 1 6}$ & $\mathbf{O 1 7}$ & $\mathbf{O 1 8}$ & $\mathbf{O 1 9}$ & $\mathbf{O 2 0}$ & $\mathbf{O 2 1}$ & $\mathbf{O 2 2}$ \\
\hline $\boldsymbol{V}_{\boldsymbol{a}}$ & 1.86 & 1.71 & 1.70 & 1.71 & 1.74 & 1.98 & 1.69 & 1.76 & 1.68 & 1.68 & 1.80 \\
\hline Site & $\mathbf{O 2 3}$ & $\mathbf{O 2 4}$ & $\mathbf{O 2 5}$ & $\mathbf{O 2 6}$ & $\mathbf{O 2 7}$ & $\mathbf{O 2 8}$ & $\mathbf{O 2 9}$ & $\mathbf{O 3 0}$ & $\mathbf{O 3 1}$ & $\mathbf{O 3 2}$ & $\mathbf{O 3 3}$ \\
\hline $\boldsymbol{V}_{\boldsymbol{a}}$ & 1.48 & 1.44 & 2.22 & 2.13 & 2.23 & 2.26 & 2.17 & 2.25 & 2.07 & 2.07 & 2.18 \\
\hline Site & $\mathbf{O 3 4}$ & $\mathbf{O 3 5}$ & $\mathbf{O 3 6}$ & $\mathbf{O 3 7}$ & $\mathbf{O 3 8}$ & $\mathbf{O 3 9}$ & $\mathbf{O 4 0}$ & $\mathbf{O 4 1}$ & $\mathbf{O 4 2}$ & $\mathbf{O 4 3}$ & $\mathbf{O 4 4}$ \\
\hline $\boldsymbol{V}_{\boldsymbol{a}}$ & 2.05 & 2.13 & 2.08 & 2.11 & 2.10 & 1.90 & 2.12 & 2.07 & 2.14 & 1.97 & 2.04 \\
\hline Site & $\mathbf{O 4 5}$ & $\mathbf{O 4 6}$ & $\mathbf{O 4 7}$ & $\mathbf{O 4 8}$ & $\mathbf{O 4 9}$ & $\mathbf{O 5 0}$ & $\mathbf{O 5 1}$ & $\mathbf{O 5 2}$ & $\mathbf{O 5 3}$ & $\mathbf{O 5 4}$ & $\mathbf{O 5 5}$ \\
\hline $\boldsymbol{V}_{\boldsymbol{a}}$ & 2.08 & 1.92 & 2.07 & 1.84 & 1.98 & 2.00 & 2.06 & 1.96 & 1.95 & 2.06 & 1.96 \\
\hline Site & $\mathbf{O 5 6}$ & $\mathbf{O 5 7}$ & $\mathbf{O 5 8}$ & $\mathbf{O 5 9}$ & $\mathbf{O 6 0}$ & $\mathbf{O 6 1}$ & $\mathbf{O 6 2}$ & $\mathbf{O 6 3}$ & $\mathbf{O 6 4}$ & $\mathbf{O 6 5}$ & $\mathbf{O 6 6}$ \\
\hline $\boldsymbol{V}_{\boldsymbol{a}}$ & 2.03 & 1.20 & 3.12 & 0.84 & 2.63 & 2.54 & 1.11 & 2.57 & 2.80 & 0.94 & 2.76 \\
\hline Site & $\mathbf{O 6 7}$ & $\mathbf{O 6 8}$ & $\mathbf{O 6 9}$ & $\mathbf{O 7 0}$ & $\mathbf{O 7 1}$ & $\mathbf{O 7 2}$ & $\mathbf{O 7 3}$ & $\mathbf{O 7 4}$ & $\mathbf{O 7 5}$ & $\mathbf{O 7 6}$ & $\mathbf{O 7 7}$ \\
\hline $\boldsymbol{V}_{\boldsymbol{a}}$ & 3.03 & 1.05 & 0.61 & 0.49 & 2.48 & 2.48 & 2.60 & 2.87 & 2.66 & 2.54 & 2.60 \\
\hline Site & $\mathbf{O 7 8}$ & & & & & & & & & & \\
\hline $\boldsymbol{V}_{\boldsymbol{a}}$ & 2.45 & & & & & & & & & & \\
\hline
\end{tabular}


Table S4. BVS for cations uranium, vanadium, and phosphorus, and BVS for oxygen anions of the structural unit in $\left\{\boldsymbol{U}_{\boldsymbol{8}} \boldsymbol{V}_{\boldsymbol{6}}\right\}$-dimer. BVS for oxygen atoms that are highlighted in orange-yellow are inferred to have a missing C(IV)-O bond, so their BVS is expectedly low. The $\mathrm{C}$ atoms were not located in the difference Fourier map. BVS for oxygen atoms that are highlighted in blue are inferred to be water oxygen atoms, missing two $\mathrm{O}-\mathrm{H}$ bonds. The $\mathrm{H}$ atoms were not located in the difference Fourier map.

\begin{tabular}{|c|c|c|c|c|c|c|c|c|}
\hline Site & $\mathbf{U 1}$ & $\mathbf{U 2}$ & $\mathbf{U 3}$ & $\mathbf{U 4}$ & $\mathbf{U 5}$ & $\mathbf{U 6}$ & $\mathbf{U 7}$ & $\mathbf{U 8}$ \\
\hline $\mathbf{O}$ & 1.78 & 1.66 & 1.67 & 1.44 & 1.75 & 1.67 & 1.64 & 1.69 \\
\hline $\mathbf{O}$ & 1.88 & 1.69 & 1.61 & 1.67 & 1.21 & 1.77 & 1.69 & 1.75 \\
\hline $\mathbf{O}$ & 0.57 & 0.55 & 0.57 & 0.57 & 0.60 & 0.61 & 0.60 & 0.58 \\
\hline $\mathbf{O}$ & 0.53 & 0.52 & 0.55 & 0.53 & 0.56 & 0.55 & 0.56 & 0.53 \\
\hline $\mathbf{O}$ & 0.58 & 0.53 & 0.56 & 0.54 & 0.62 & 0.56 & 0.52 & 0.53 \\
\hline $\mathbf{O}$ & 0.57 & 0.55 & 0.53 & 0.50 & 0.39 & 0.56 & 0.56 & 0.58 \\
\hline $\mathbf{O}$ & 0.54 & 0.54 & 0.61 & 0.56 & 0.53 & 0.56 & 0.55 & 0.60 \\
\hline $\boldsymbol{V}_{\boldsymbol{c}}$ & 6.45 & 6.06 & 6.10 & 5.82 & 5.67 & 6.28 & 6.11 & 6.26 \\
\hline
\end{tabular}

\begin{tabular}{|c|c|c|c|c|c|c|}
\hline Site & $\mathbf{V 1}$ & $\mathbf{V 2}$ & $\mathbf{V 3}$ & $\mathbf{V 4}$ & $\mathbf{V 5}$ & $\mathbf{V 6}$ \\
\hline $\mathbf{O}$ & 1.70 & 1.77 & 1.71 & 1.74 & 1.71 & 1.85 \\
\hline $\mathbf{O}$ & 0.73 & 0.69 & 0.83 & 0.82 & 0.90 & 0.72 \\
\hline $\mathbf{O}$ & 0.70 & 0.93 & 0.81 & 0.78 & 0.84 & 0.74 \\
\hline $\mathbf{O}$ & 1.05 & 0.78 & 0.84 & 0.92 & 0.83 & 0.94 \\
\hline $\mathbf{O}$ & 1.01 & 0.97 & 0.91 & 1.00 & 0.86 & 1.07 \\
\hline $\boldsymbol{V}_{\boldsymbol{c}}$ & 5.19 & 5.14 & 5.11 & 5.26 & 5.14 & 5.33 \\
\hline
\end{tabular}




\begin{tabular}{|c|c|c|c|c|c|c|c|c|c|}
\hline Site & P1 & P2 & P3 & P4 & P5 & P6 & P7 & P8 & P9 \\
\hline $\mathbf{O}$ & 0.99 & 1.41 & 1.46 & 1.53 & 1.30 & 1.80 & 1.32 & 1.37 & 1.43 \\
\hline $\mathbf{O}$ & 0.97 & 1.55 & 1.57 & 1.49 & 1.80 & 1.41 & 1.38 & 1.48 & 1.41 \\
\hline $\mathbf{O}$ & 1.08 & 1.37 & 1.08 & 1.11 & 1.02 & 1.27 & 1.30 & 1.20 & 1.08 \\
\hline $\mathbf{O}$ & 1.07 & 1.08 & 1.08 & 1.06 & 0.58 & 1.08 & 1.57 & 1.34 & 1.07 \\
\hline $\boldsymbol{V}_{\boldsymbol{c}}$ & 4.10 & 5.41 & 5.19 & 5.18 & 4.69 & 5.55 & 5.57 & 5.39 & 4.98 \\
\hline
\end{tabular}

\begin{tabular}{|c|c|c|c|c|c|c|c|c|c|c|c|}
\hline Site & $\mathbf{O 1}$ & $\mathbf{O 2}$ & $\mathbf{O 3}$ & $\mathbf{O 4}$ & $\mathbf{O 5}$ & $\mathbf{O 6}$ & $\mathbf{O 7}$ & $\mathbf{O 8}$ & $\mathbf{O 9}$ & $\mathbf{O 1 0}$ & $\mathbf{O 1 1}$ \\
\hline $\boldsymbol{V}_{\boldsymbol{a}}$ & 1.78 & 1.88 & 1.66 & 1.69 & 1.67 & 1.61 & 1.44 & 1.67 & 1.75 & 1.21 & 1.67 \\
\hline Site & $\mathbf{O 1 2}$ & $\mathbf{O 1 3}$ & $\mathbf{O 1 4}$ & $\mathbf{O 1 5}$ & $\mathbf{O 1 6}$ & $\mathbf{O 1 7}$ & $\mathbf{O 1 8}$ & $\mathbf{O 1 9}$ & $\mathbf{O 2 0}$ & $\mathbf{O 2 1}$ & $\mathbf{O 2 2}$ \\
\hline $\boldsymbol{V}_{\boldsymbol{a}}$ & 1.77 & 1.64 & 1.69 & 1.69 & 1.75 & 1.70 & 1.77 & 1.71 & 1.74 & 1.71 & 1.85 \\
\hline Site & $\mathbf{O 2 3}$ & $\mathbf{O 2 4}$ & $\mathbf{O 2 5}$ & $\mathbf{O 2 6}$ & $\mathbf{O 2 7}$ & $\mathbf{O 2 8}$ & $\mathbf{O 2 9}$ & $\mathbf{O 3 0}$ & $\mathbf{O 3 1}$ & $\mathbf{O 3 2}$ & $\mathbf{O 3 3}$ \\
\hline $\boldsymbol{V}_{\boldsymbol{a}}$ & 1.42 & 1.72 & 2.22 & 2.16 & 2.24 & 2.23 & 2.17 & 2.22 & 2.14 & 2.05 & 2.01 \\
\hline Site & $\mathbf{O 3 4}$ & $\mathbf{O 3 5}$ & $\mathbf{O 3 6}$ & $\mathbf{O 3 7}$ & $\mathbf{O 3 8}$ & $\mathbf{O 3 9}$ & $\mathbf{O 4 0}$ & $\mathbf{O 4 1}$ & $\mathbf{O 4 2}$ & $\mathbf{O 4 3}$ & $\mathbf{O 4 4}$ \\
\hline $\boldsymbol{V}_{\boldsymbol{a}}$ & 2.06 & 2.12 & 1.98 & 2.12 & 2.07 & 1.55 & 1.98 & 2.10 & 2.01 & 2.10 & 2.14 \\
\hline Site & $\mathbf{O 4 5}$ & $\mathbf{O 4 6}$ & $\mathbf{O 4 7}$ & $\mathbf{O 4 8}$ & $\mathbf{O 4 9}$ & $\mathbf{O 5 0}$ & $\mathbf{O 5 1}$ & $\mathbf{O 5 2}$ & $\mathbf{O 5 3}$ & $\mathbf{O 5 4}$ & $\mathbf{O 5 5}$ \\
\hline $\boldsymbol{V}_{\boldsymbol{a}}$ & 1.99 & 1.86 & 2.42 & 0.39 & 2.33 & 1.97 & 1.88 & 1.94 & 1.92 & 2.06 & 2.03 \\
\hline Site & $\mathbf{O 5 6}$ & $\mathbf{O 5 7}$ & $\mathbf{O 5 8}$ & $\mathbf{O 5 9}$ & $\mathbf{O 6 0}$ & $\mathbf{O 6 1}$ & $\mathbf{O 6 2}$ & $\mathbf{O 6 3}$ & $\mathbf{O 6 4}$ & $\mathbf{O 6 5}$ & $\mathbf{O 6 6}$ \\
\hline $\boldsymbol{V}_{\boldsymbol{a}}$ & 1.95 & 1.90 & 1.89 & 2.20 & 1.08 & 1.90 & 1.08 & 1.11 & 1.88 & 1.02 & 0.58 \\
\hline Site & $\mathbf{O 6 7}$ & $\mathbf{O 6 8}$ & $\mathbf{O 6 9}$ & $\mathbf{O 7 0}$ & $\mathbf{O 7 1}$ & $\mathbf{O 7 2}$ & $\mathbf{O 7 3}$ & $\mathbf{O 7 4}$ & & & \\
\hline $\boldsymbol{V}_{\boldsymbol{a}}$ & 1.93 & 1.91 & 2.13 & 2.40 & 1.20 & 1.34 & 1.08 & 1.72 & & & \\
\hline
\end{tabular}


Table S5. BVS for cations uranium, vanadium, and phosphorus, and BVS for anionic oxygen atoms of the structural unit in $\left\{\boldsymbol{U}_{\boldsymbol{8}} \boldsymbol{V}_{\boldsymbol{b}}\right\}-$

chain.

\begin{tabular}{|c|c|c|c|c|c|c|c|c|}
\hline Site & $\mathbf{U 1}$ & $\mathbf{U 2}$ & $\mathbf{U 3}$ & $\mathbf{U 4}$ & $\mathbf{U 5}$ & $\mathbf{U 6}$ & $\mathbf{U 7}$ & $\mathbf{U 8}$ \\
\hline $\mathbf{O}$ & 1.72 & 1.68 & 1.66 & 1.68 & 1.66 & 1.67 & 1.65 & 1.69 \\
\hline $\mathbf{O}$ & 1.71 & 1.69 & 1.67 & 1.68 & 1.71 & 1.63 & 1.67 & 1.66 \\
\hline $\mathbf{O}$ & 0.60 & 0.59 & 0.59 & 0.59 & 0.58 & 0.61 & 0.59 & 0.59 \\
\hline $\mathbf{O}$ & 0.55 & 0.54 & 0.56 & 0.58 & 0.52 & 0.57 & 0.59 & 0.57 \\
\hline $\mathbf{O}$ & 0.54 & 0.53 & 0.56 & 0.56 & 0.51 & 0.53 & 0.54 & 0.55 \\
\hline $\mathbf{O}$ & 0.51 & 0.52 & 0.53 & 0.55 & 0.51 & 0.53 & 0.54 & 0.53 \\
\hline $\mathbf{O}$ & 0.50 & 0.51 & 0.53 & 0.53 & 0.50 & 0.53 & 0.52 & 0.53 \\
\hline $\boldsymbol{V}_{\boldsymbol{c}}$ & 6.11 & 6.06 & 6.09 & 6.16 & 5.99 & 6.07 & 6.08 & 6.10 \\
\hline
\end{tabular}

\begin{tabular}{|c|c|c|c|c|c|c|}
\hline Site & $\mathbf{V 1}$ & $\mathbf{V 2}$ & $\mathbf{V 3}$ & $\mathbf{V 4}$ & $\mathbf{V 5}$ & $\mathbf{V 6}$ \\
\hline $\mathbf{O}$ & 1.64 & 1.75 & 1.74 & 1.75 & 1.76 & 1.70 \\
\hline $\mathbf{O}$ & 0.98 & 0.93 & 1.01 & 0.99 & 0.98 & 0.88 \\
\hline $\mathbf{O}$ & 0.92 & 0.93 & 0.95 & 0.95 & 0.88 & 0.87 \\
\hline $\mathbf{O}$ & 0.76 & 0.87 & 0.86 & 0.77 & 0.85 & 0.86 \\
\hline $\mathbf{O}$ & 0.70 & 0.70 & 0.68 & 0.69 & 0.65 & 0.73 \\
\hline $\boldsymbol{V}_{\boldsymbol{c}}$ & 5.00 & 5.19 & 5.24 & 5.15 & 5.11 & 5.03 \\
\hline
\end{tabular}

\begin{tabular}{|c|c|c|c|c|c|c|c|c|c|}
\hline Site & P1 & P2 & P3 & P4 & P5 & P6 & P7 & P8 & P9 \\
\hline $\mathbf{O}$ & 1.42 & 1.41 & 1.42 & 1.41 & 1.39 & 1.46 & 1.44 & 1.37 & 1.48 \\
\hline $\mathbf{O}$ & 1.38 & 1.38 & 1.42 & 1.40 & 1.29 & 1.36 & 1.41 & 1.37 & 1.40 \\
\hline $\mathbf{O}$ & 1.09 & 1.15 & 1.16 & 1.20 & 1.14 & 1.11 & 1.14 & 1.14 & 1.14 \\
\hline $\mathbf{O}$ & 1.09 & 1.07 & 1.11 & 1.12 & 1.10 & 1.10 & 1.09 & 1.11 & 1.09 \\
\hline $\boldsymbol{V}_{\boldsymbol{c}}$ & 4.98 & 5.01 & 5.11 & 5.12 & 4.93 & 5.04 & 5.08 & 4.99 & 5.11 \\
\hline
\end{tabular}




\begin{tabular}{|c|c|c|c|c|c|c|c|c|c|c|c|}
\hline Site & $\mathbf{O 1}$ & $\mathbf{O 2}$ & $\mathbf{O 3}$ & $\mathbf{O 4}$ & $\mathbf{O 5}$ & $\mathbf{O 6}$ & $\mathbf{O 7}$ & $\mathbf{O 8}$ & $\mathbf{O 9}$ & $\mathbf{O 1 0}$ & $\mathbf{O 1 1}$ \\
\hline $\boldsymbol{V}_{\boldsymbol{a}}$ & 1.72 & 1.71 & 1.68 & 1.69 & 1.66 & 1.67 & 1.68 & 1.68 & 1.66 & 1.71 & 1.67 \\
\hline Site & $\mathbf{O 1 2}$ & $\mathbf{O 1 3}$ & $\mathbf{O 1 4}$ & $\mathbf{O 1 5}$ & $\mathbf{O 1 6}$ & $\mathbf{O 1 7}$ & $\mathbf{O 1 8}$ & $\mathbf{O 1 9}$ & $\mathbf{O 2 0}$ & $\mathbf{O 2 1}$ & $\mathbf{O 2 2}$ \\
\hline $\boldsymbol{V}_{\boldsymbol{a}}$ & 1.63 & 1.65 & 1.67 & 1.66 & 1.69 & 1.64 & 1.75 & 1.74 & 1.75 & 1.76 & 1.70 \\
\hline Site & $\mathbf{O 2 3}$ & $\mathbf{O 2 4}$ & $\mathbf{O 2 5}$ & $\mathbf{O 2 6}$ & $\mathbf{O 2 7}$ & $\mathbf{O 2 8}$ & $\mathbf{O 2 9}$ & $\mathbf{O 3 0}$ & $\mathbf{O 3 1}$ & $\mathbf{O 3 2}$ & $\mathbf{O 3 3}$ \\
\hline $\boldsymbol{V}_{\boldsymbol{a}}$ & 1.73 & 1.32 & 2.23 & 2.15 & 2.24 & 2.26 & 2.16 & 2.22 & 2.03 & 2.02 & 2.10 \\
\hline Site & $\mathbf{O 3 4}$ & $\mathbf{O 3 5}$ & $\mathbf{O 3 6}$ & $\mathbf{O 3 7}$ & $\mathbf{O 3 8}$ & $\mathbf{O 3 9}$ & $\mathbf{O 4 0}$ & $\mathbf{O 4 1}$ & $\mathbf{O 4 2}$ & $\mathbf{O 4 3}$ & $\mathbf{O 4 4}$ \\
\hline $\boldsymbol{V}_{\boldsymbol{a}}$ & 2.10 & 2.03 & 2.05 & 2.03 & 2.04 & 1.92 & 1.96 & 1.92 & 1.95 & 1.95 & 1.94 \\
\hline Site & $\mathbf{O 4 5}$ & $\mathbf{O 4 6}$ & $\mathbf{O 4 7}$ & $\mathbf{O 4 8}$ & $\mathbf{O 4 9}$ & $\mathbf{O 5 0}$ & $\mathbf{O 5 2}$ & $\mathbf{O 5 3}$ & $\mathbf{O 5 4}$ & $\mathbf{O 5 5}$ & $\mathbf{O 5 6}$ \\
\hline $\boldsymbol{V}_{\boldsymbol{a}}$ & 1.95 & 1.85 & 1.91 & 1.90 & 1.96 & 1.93 & 1.94 & 1.97 & 1.88 & 1.90 & 1.95 \\
\hline Site & $\mathbf{O 5 7}$ & $\mathbf{O 5 8}$ & $\mathbf{O 5 9}$ & $\mathbf{O 6 0}$ & $\mathbf{O 6 1}$ & $\mathbf{O 6 2}$ & $\mathbf{O 6 3}$ & $\mathbf{O 6 4}$ & $\mathbf{O 6 5}$ & $\mathbf{O 6 6}$ & $\mathbf{O 6 7}$ \\
\hline $\boldsymbol{V}_{\boldsymbol{a}}$ & 1.99 & 2.03 & 1.92 & 1.93 & 2.01 & 2.03 & 1.94 & 2.01 & 1.98 & 1.89 & 2.14 \\
\hline Site & $\mathbf{O 6 8}$ & $\mathbf{O 6 9}$ & $\mathbf{O 7 0}$ & $\mathbf{O 7 1}$ & $\mathbf{O 7 2}$ & $\mathbf{O 7 3}$ & $\mathbf{O 7 4}$ & $\mathbf{O 7 5}$ & & & \\
\hline $\boldsymbol{V}_{\boldsymbol{a}}$ & 1.96 & 2.00 & 1.93 & 1.97 & 1.93 & 2.14 & 1.91 & 1.96 & & & \\
\hline
\end{tabular}


Table S6. BVS for cations uranium, vanadium, tungsten, and phosphorus, and BVS for anionic oxygen atoms of the structural unit in $\left\{U_{6} V_{3}\right\}-W$

\begin{tabular}{|c|c|c|c|c|c|c|}
\hline Site & $\mathbf{U 1}$ & $\mathbf{U 2}$ & $\mathbf{U 3}$ & $\mathbf{U 4}$ & $\mathbf{U 5}$ & $\mathbf{U 6}$ \\
\hline $\mathbf{O}$ & 1.70 & 1.70 & 1.71 & 1.68 & 1.73 & 1.70 \\
\hline $\mathbf{O}$ & 1.70 & 1.64 & 1.70 & 1.65 & 1.69 & 1.69 \\
\hline $\mathbf{O}$ & 0.61 & 0.60 & 0.54 & 0.61 & 0.55 & 0.57 \\
\hline $\mathbf{O}$ & 0.56 & 0.58 & 0.53 & 0.58 & 0.54 & 0.56 \\
\hline $\mathbf{O}$ & 0.53 & 0.56 & 0.53 & 0.58 & 0.53 & 0.55 \\
\hline $\mathbf{O}$ & 0.52 & 0.51 & 0.53 & 0.49 & 0.53 & 0.51 \\
\hline $\mathbf{O}$ & 0.52 & 0.51 & 0.52 & 0.49 & 0.53 & 0.49 \\
\hline $\boldsymbol{V}_{\boldsymbol{c}}$ & 6.15 & 6.10 & 6.05 & 6.08 & 6.09 & 6.07 \\
\hline
\end{tabular}

\begin{tabular}{|c|c|c|c|c|c|c|}
\hline Site & $\mathbf{W 1}$ & $\mathbf{W 2}$ & $\mathbf{W 3}$ & $\mathbf{V 1}$ & $\mathbf{V 2}$ & $\mathbf{V 3}$ \\
\hline $\mathbf{O}$ & 2.34 & 2.47 & 2.17 & 1.74 & 1.84 & 1.61 \\
\hline $\mathbf{O}$ & 1.19 & 1.22 & 1.16 & 0.88 & 0.90 & 0.86 \\
\hline $\mathbf{O}$ & 1.17 & 1.21 & 1.15 & 0.87 & 0.90 & 0.85 \\
\hline $\mathbf{O}$ & 0.95 & 0.98 & 1.02 & 0.70 & 0.72 & 0.75 \\
\hline $\mathbf{O}$ & 0.93 & 0.97 & 1.00 & 0.69 & 0.72 & 0.74 \\
\hline $\boldsymbol{V}_{\boldsymbol{c}}$ & 6.57 & 6.84 & 6.49 & 4.88 & 5.08 & 4.82 \\
\hline
\end{tabular}

\begin{tabular}{|c|c|c|c|c|c|c|c|c|}
\hline Site & P1 & P2 & P3 & P4 & P5 & P6 & P7 & P8 \\
\hline $\mathbf{O}$ & 1.52 & 1.43 & 1.47 & 1.39 & 1.45 & 1.40 & 1.50 & 1.43 \\
\hline $\mathbf{O}$ & 1.31 & 1.42 & 1.39 & 1.36 & 1.42 & 1.39 & 1.42 & 1.40 \\
\hline $\mathbf{O}$ & 1.26 & 1.19 & 1.22 & 1.14 & 1.23 & 1.15 & 1.36 & 1.15 \\
\hline $\mathbf{O}$ & 1.21 & 1.14 & 1.02 & 1.07 & 0.99 & 1.14 & 1.17 & 1.15 \\
\hline $\boldsymbol{V}_{\boldsymbol{c}}$ & 5.30 & 5.19 & 5.10 & 4.96 & 5.08 & 5.07 & 5.45 & 5.14 \\
\hline
\end{tabular}




\begin{tabular}{|c|c|c|c|c|c|c|c|c|c|c|c|}
\hline Site & $\mathbf{O 1}$ & $\mathbf{O 2}$ & $\mathbf{O 3}$ & $\mathbf{O 4}$ & $\mathbf{O 5}$ & $\mathbf{O 6}$ & $\mathbf{O 7}$ & $\mathbf{O 8}$ & $\mathbf{O 9}$ & $\mathbf{O 1 0}$ & $\mathbf{O 1 1}$ \\
\hline $\boldsymbol{V}_{\boldsymbol{a}}$ & 1.70 & 1.70 & 1.64 & 1.70 & 1.70 & 1.71 & 1.68 & 1.65 & 1.73 & 1.69 & 1.69 \\
\hline Site & $\mathbf{O 1 2}$ & $\mathbf{O 1 3}$ & $\mathbf{O 1 4}$ & $\mathbf{O 1 5}$ & $\mathbf{O 1 6}$ & $\mathbf{O 1 7}$ & $\mathbf{O 1 8}$ & $\mathbf{O 1 9}$ & $\mathbf{O 2 0}$ & $\mathbf{O 2 1}$ & $\mathbf{O 2 2}$ \\
\hline $\boldsymbol{V}_{\boldsymbol{a}}$ & 1.70 & 1.74 & 1.84 & 1.61 & 2.00 & 2.06 & 2.00 & 1.91 & 1.93 & 1.93 & 1.87 \\
\hline Site & $\mathbf{O 2 3}$ & $\mathbf{O 2 4}$ & $\mathbf{O 2 5}$ & $\mathbf{O 2 6}$ & $\mathbf{O 2 7}$ & $\mathbf{O 2 8}$ & $\mathbf{O 2 9}$ & $\mathbf{O 3 0}$ & $\mathbf{O 3 1}$ & $\mathbf{O 3 2}$ & $\mathbf{O 3 3}$ \\
\hline $\boldsymbol{V}_{\boldsymbol{a}}$ & 1.87 & 1.91 & 1.92 & 1.99 & 1.99 & 1.99 & 2.00 & 1.90 & 1.98 & 2.03 & 1.98 \\
\hline Site & $\mathbf{O 3 4}$ & $\mathbf{O 3 5}$ & $\mathbf{O 3 6}$ & $\mathbf{O 3 7}$ & $\mathbf{O 3 8}$ & $\mathbf{O 3 9}$ & $\mathbf{O 4 0}$ & $\mathbf{O 4 1}$ & $\mathbf{O 4 2}$ & $\mathbf{O 4 3}$ & $\mathbf{O 4 4}$ \\
\hline $\boldsymbol{V}_{\boldsymbol{a}}$ & 1.93 & 1.94 & 2.02 & 2.00 & 1.95 & 1.96 & 2.42 & 2.51 & 1.52 & 2.10 & 2.10 \\
\hline Site & $\mathbf{O 4 5}$ & $\mathbf{O 4 6}$ & $\mathbf{O 4 7}$ & $\mathbf{O 4 8}$ & $\mathbf{O 4 9}$ & $\mathbf{O 5 0}$ & $\mathbf{O 5 1}$ & $\mathbf{O 5 2}$ & $\mathbf{O 5 3}$ & $\mathbf{O 5 4}$ & $\mathbf{O 5 5}$ \\
\hline $\boldsymbol{V}_{\boldsymbol{a}}$ & 1.76 & 2.31 & 1.99 & 2.22 & 1.52 & 2.08 & 2.01 & 2.03 & 3.14 & 2.03 & 1.94 \\
\hline Site & $\mathbf{O 5 6}$ & & & & & & & & & & \\
\hline $\boldsymbol{V}_{\boldsymbol{a}}$ & 1.99 & & & & & & & & & & \\
\hline
\end{tabular}


Table S7. Bond valence summations (BVS) for vanadium and oxygen in $\left\{\boldsymbol{V}_{10}\right\}$ POM side-product. The bond valence summations of the oxygen anions highlighted in green are inferred to be hydroxide oxygen atoms.

\begin{tabular}{|c|c|c|c|c|c|c|c|c|c|c|}
\hline Site & $\mathbf{V 1}$ & $\mathbf{V 2}$ & $\mathbf{V 3}$ & $\mathbf{V 4}$ & $\mathbf{V 5}$ & $\mathbf{V 6}$ & $\mathbf{V 7}$ & $\mathbf{V 8}$ & $\mathbf{V 9}$ & $\mathbf{V 1 0}$ \\
\hline $\mathbf{O}$ & 0.35 & 0.31 & 0.23 & 0.40 & 0.20 & 0.22 & 0.42 & 0.29 & 0.24 & 0.27 \\
\hline $\mathbf{O}$ & 1.23 & 0.44 & 0.64 & 0.48 & 0.73 & 0.83 & 0.51 & 0.59 & 1.00 & 0.55 \\
\hline $\mathbf{O}$ & 1.57 & 0.56 & 0.96 & 0.67 & 0.53 & 0.85 & 0.52 & 0.89 & 0.64 & 1.02 \\
\hline $\mathbf{O}$ & 0.67 & 1.56 & 0.51 & 1.31 & 1.73 & 1.70 & 1.34 & 0.62 & 0.55 & 1.01 \\
\hline $\mathbf{O}$ & 0.39 & 1.17 & 0.90 & 1.34 & 0.93 & 0.83 & 1.33 & 1.73 & 0.87 & 0.57 \\
\hline $\mathbf{O}$ & 0.74 & 1.01 & 1.69 & 0.75 & 1.02 & 0.49 & 0.87 & 1.07 & 1.76 & 1.63 \\
\hline $\boldsymbol{V}_{\boldsymbol{c}}$ & 4.94 & 5.05 & 4.93 & 4.95 & 5.13 & 4.94 & 4.99 & 5.18 & 5.06 & 5.04 \\
\hline
\end{tabular}

\begin{tabular}{|c|c|c|c|c|c|c|c|c|c|c|}
\hline Site & $\mathbf{V 1 1}$ & $\mathbf{V 1 2}$ & $\mathbf{V 1 3}$ & $\mathbf{V 1 4}$ & $\mathbf{V 1 5}$ & $\mathbf{V 1 6}$ & $\mathbf{V 1 7}$ & $\mathbf{V 1 8}$ & $\mathbf{V 1 9}$ & $\mathbf{V 2 0}$ \\
\hline $\mathbf{O}$ & 0.25 & 0.23 & 0.19 & 0.49 & 0.23 & 0.29 & 0.49 & 0.22 & 0.32 & 0.36 \\
\hline $\mathbf{O}$ & 1.74 & 0.56 & 0.96 & 0.39 & 0.86 & 0.99 & 0.43 & 0.84 & 1.10 & 0.75 \\
\hline $\mathbf{O}$ & 0.58 & 0.66 & 1.79 & 0.77 & 0.51 & 0.88 & 0.88 & 0.92 & 0.53 & 1.24 \\
\hline $\mathbf{O}$ & 0.52 & 1.68 & 0.94 & 1.38 & 1.02 & 1.70 & 1.40 & 0.52 & 0.44 & 0.68 \\
\hline $\mathbf{O}$ & 0.91 & 0.91 & 0.51 & 0.66 & 0.63 & 0.55 & 1.30 & 1.66 & 1.02 & 0.42 \\
\hline $\mathbf{O}$ & 0.99 & 1.03 & 0.75 & 1.25 & 1.71 & 0.55 & 0.50 & 0.82 & 1.69 & 1.51 \\
\hline $\boldsymbol{V}_{\boldsymbol{c}}$ & 4.99 & 5.06 & 5.13 & 4.94 & 4.96 & 4.97 & 4.99 & 4.98 & 5.11 & 4.98 \\
\hline
\end{tabular}




\begin{tabular}{|c|c|c|c|c|c|c|c|c|c|c|c|}
\hline Site & $\mathbf{O 1}$ & $\mathbf{O 2}$ & $\mathbf{O 3}$ & $\mathbf{O 4}$ & $\mathbf{O 5}$ & $\mathbf{O 6}$ & $\mathbf{O 7}$ & $\mathbf{O 8}$ & $\mathbf{O 9}$ & $\mathbf{O 1 0}$ & $\mathbf{O 1 1}$ \\
\hline $\boldsymbol{V}_{\boldsymbol{a}}$ & 1.92 & 1.99 & 1.87 & 1.57 & 1.26 & 1.34 & 1.96 & 1.56 & 1.90 & 1.84 & 1.85 \\
\hline Site & $\mathbf{O 1 2}$ & $\mathbf{O 1 3}$ & $\mathbf{O 1 4}$ & $\mathbf{O 1 5}$ & $\mathbf{O 1 6}$ & $\mathbf{O 1 7}$ & $\mathbf{O 1 8}$ & $\mathbf{O 1 9}$ & $\mathbf{O 2 0}$ & $\mathbf{O 2 1}$ & $\mathbf{O 2 2}$ \\
\hline $\boldsymbol{V}_{\boldsymbol{a}}$ & 1.82 & 1.90 & 1.69 & 1.87 & 1.94 & 1.73 & 1.96 & 1.87 & 1.70 & 1.84 & 1.84 \\
\hline Site & $\mathbf{O 2 3}$ & $\mathbf{O 2 4}$ & $\mathbf{O 2 5}$ & $\mathbf{O 2 6}$ & $\mathbf{O 2 7}$ & $\mathbf{O 2 8}$ & $\mathbf{O 2 9}$ & $\mathbf{O 3 0}$ & $\mathbf{O 3 1}$ & $\mathbf{O 3 2}$ & $\mathbf{O 3 3}$ \\
\hline $\boldsymbol{V}_{\boldsymbol{a}}$ & 1.95 & 1.99 & 1.73 & 1.93 & 1.76 & 1.63 & 1.97 & 1.93 & 1.74 & 2.02 & 1.95 \\
\hline Site & $\mathbf{O 3 4}$ & $\mathbf{O 3 5}$ & $\mathbf{O 3 6}$ & $\mathbf{O 3 7}$ & $\mathbf{O 3 8}$ & $\mathbf{O 3 9}$ & $\mathbf{O 4 0}$ & $\mathbf{O 4 1}$ & $\mathbf{O 4 2}$ & $\mathbf{O 4 3}$ & $\mathbf{O 4 4}$ \\
\hline $\boldsymbol{V}_{\boldsymbol{a}}$ & 1.87 & 1.83 & 1.68 & 1.90 & 1.89 & 1.79 & 1.86 & 1.89 & 1.85 & 1.94 & 1.76 \\
\hline Site & $\mathbf{O 4 5}$ & $\mathbf{O 4 6}$ & $\mathbf{O 4 7}$ & $\mathbf{O 4 8}$ & $\mathbf{O 4 9}$ & $\mathbf{O 5 0}$ & $\mathbf{O 5 1}$ & $\mathbf{O 5 2}$ & $\mathbf{O 5 3}$ & $\mathbf{O 5 4}$ & $\mathbf{O 5 5}$ \\
\hline $\boldsymbol{V}_{\boldsymbol{a}}$ & 1.90 & 1.87 & 1.71 & 1.70 & 1.95 & 1.24 & 1.82 & 1.36 & 1.66 & 1.84 & 1.69 \\
\hline Site & $\mathbf{O 5 6}$ & & & & & & & & & & \\
\hline $\boldsymbol{V}_{\boldsymbol{a}}$ & 1.51 & & & & & & & & & & \\
\hline
\end{tabular}

